\title{
Momentum and scalar transport at the turbulent/non-turbulent interface of a jet
}

\author{
J. WESTER WEEL $L^{1} \dagger$, C. FUKUSHIMA ${ }^{2}$, \\ J. M. PEDERSEN ${ }^{3}$ AND J. C. R. HUN T \\ ${ }^{1}$ J. M. Burgers Centre for Fluid Dynamics, Delft University of Technology, Mekelweg 2, \\ 2628 CD Delft, The Netherlands \\ ${ }^{2}$ Department of Mechanical Systems Engineering, Hiroshima Institute of Technology, \\ Miyake 2-1-1, Saeki-ku, 731-5193 Hiroshima, Japan \\ ${ }^{3}$ Department of Mechanical Engineering, Technical University of Denmark, Akademivej, \\ Bldg. 358, DK-2800 Lyngby, Denmark
}

(Received 8 March 2008 and in revised form 10 February 2009)

Conditionally sampled measurements with particle image velocimetry (PIV) of a turbulent round submerged liquid jet in a laboratory have been taken at $R e=2 \times 10^{3}$ between 60 and 100 nozzle diameters from the nozzle in order to investigate the dynamics and transport processes at the continuous and well-defined bounding interface between the turbulent and non-turbulent regions of flow. The jet carries a fluorescent dye measured with planar laser-induced fluorescence (LIF), and the surface discontinuity in the scalar concentration is identified as the fluctuating turbulent jet interface. Thence the mean outward 'boundary entrainment' velocity is derived and shown to be a constant fraction (about 0.07 ) of the the mean jet velocity on the centreline. Profiles of the conditional mean velocity, mean scalar and momentum flux show that at the interface there are clear discontinuities in the mean axial velocity and mean scalar and a tendency towards a singularity in mean vorticity. These actual or asymptotic discontinuities are consistent with the conditional mean momentum and scalar transport equations integrated across the interface. Measurements of the fluxes of turbulent kinetic energy and enstrophy are consistent with computations by Mathew \& Basu (Phys. Fluids, vol. 14, 2002, pp. 2065-2072) in showing that for a jet flow (without forcing) the entrainment process is dominated by small-scale eddying at the highly sheared interface ('nibbling'), with large-scale engulfing making a small (less than 10\%) contribution consistent with concentration measurements showing that the interior of the jet is well mixed. (Turbulent jets differ greatly from the free shear layer in this respect.) To explain the difference between velocity and scalar profiles, their conditional mean gradients are defined in terms of a local eddy viscosity and eddy diffusivity and the momentum and scalar fluxes inside the interface. Since the eddy diffusivity is larger than the eddy viscosity, the scalar profile is flatter inside the interface so that the scalar discontinuity is relatively greater than the mean velocity discontinuity. Theoretical arguments, following Hunt, Eames \& Westerweel (in Proc. of the IUTAM Symp. on Computational Physics and New Perspectives in Turbulence, ed. Y. Kaneda, vol. 4, 2008, pp. 331-338, Springer), are proposed for how the vortex sheet develops, how the internal structure of the interface layer relates to the inhomogeneous rotational and irrotational motions on each side and why the dominant entrainment process of jets and wakes differs from that of free shear layers. 


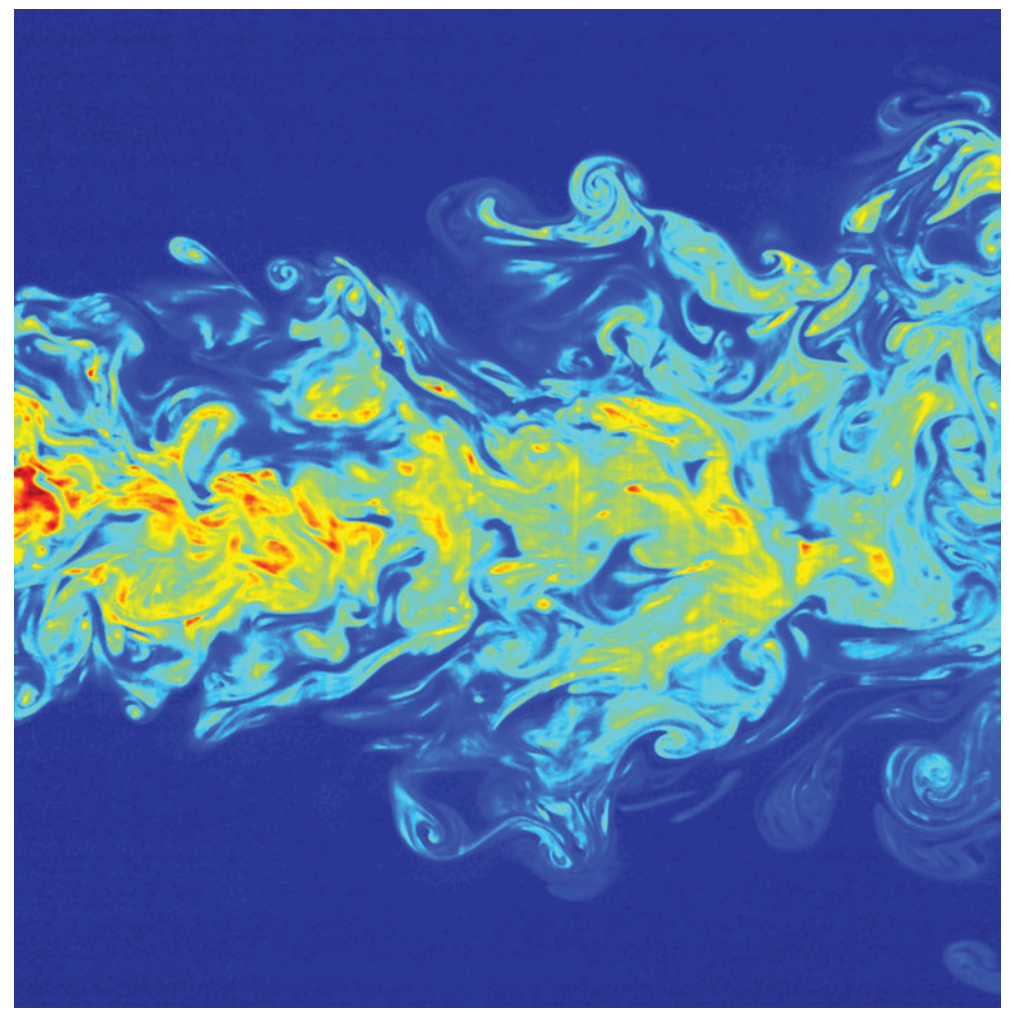

FIGURE 1. The instantaneous concentration field in the far field of a submerged self-similar turbulent jet.

\section{Introduction}

A long-standing question about unconfined, but localized, turbulent flows, such as the turbulent jet in figure 1 , is to describe and quantify the characteristic features of the inhomogeneous transition zone between fully developed turbulence and the decaying fluctuations outside the turbulent region (Hinze 1975; Townsend 1976; Hunt et al. 2001; Tsinober 2001). Recent investigations have shown that this zone generally consists of three layers: a fluctuating, but well-defined, interface (i) that is very thin (with a thickness $\ell_{\mathrm{i}}$ ) and at which the vortical fluctuations decrease rapidly. This separates the external layer $(\mathscr{E})$ of irrotational fluctuations outside the interface from the adjustment layer $(\mathscr{A})$ of inhomogeneous turbulence inside the interface in which the rotational and irrotational velocity fields adjust to their more homogeneous structure in the fully developed turbulent region (see figure 2). Typically the thicknesses of the latter layers are of the order of the integral scale $L_{x}$ of the turbulence.

This general form of the structure has been suggested by previous experiments and theoretical studies (e.g. Corrsin \& Kistler 1955; Phillips 1955; Townsend 1976; Carruthers \& Hunt 1986). Because of the lack of detailed experimental data, especially of the velocity field on either side of the fluctuating interface, theories and concepts about these transition zones have not been thoroughly tested, in particular profiles and conditional statistics of the flow field near the interface, the vortical dynamics and possible instability mechanisms of the interfacial flows (e.g. Reynolds 1972) and statistical relations between these local flows and those of the flow as a whole, such 
(a)
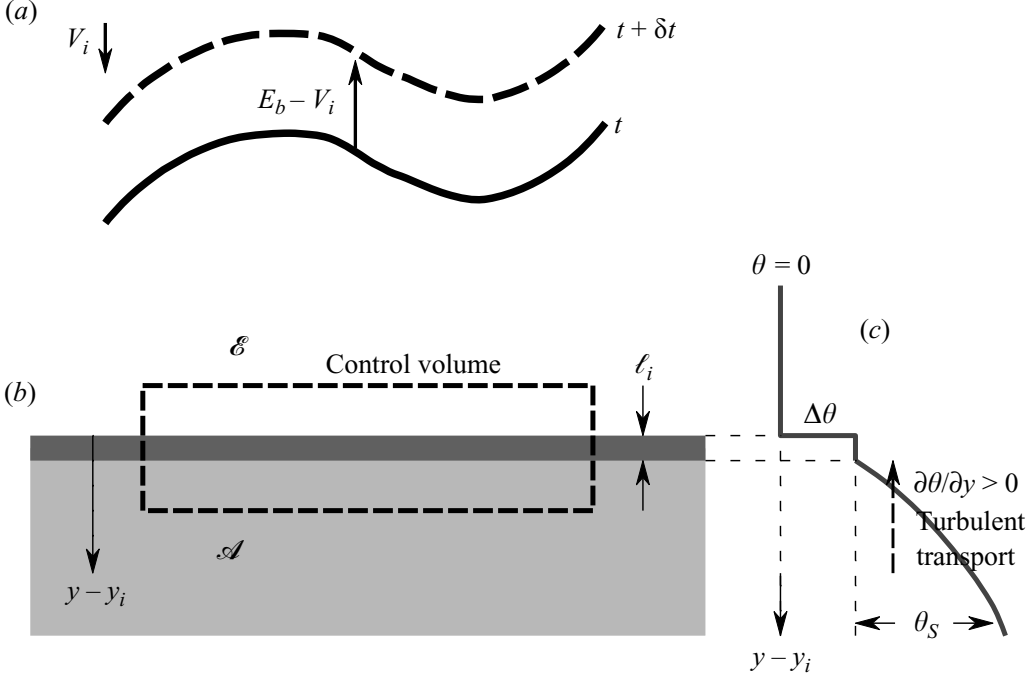

FiguRE 2. The superlayer jump condition at the turbulent/non-turbulent interface. $(a)$ The moving interface; $(b)$ definition of the interface of thickness $\ell_{\mathrm{i}}$ between the external $(\mathscr{E})$ layer and adjustment $(\mathscr{A})$ layer; $(c)$ typical mean conditional scalar profile relative to the interface (for $S c \gg 1$ ).

as the average movement of the interface and the entrainment of external flow into the turbulent region.

Brown \& Roshko (1974) showed that large-scale organized structures exist in the instantaneous flow of a turbulent plane shear layer, where the large-scale eddies draw external irrotational fluid directly into the interior of the spreading turbulent flow region. This was generally considered to be the principal mechanism for entrainment of the bounding irrotational fluid into the turbulent flow region for all free turbulent shear flows. Many experimental studies have concluded that mixing and entrainment in jets and wakes are dominated by the engulfment process (e.g. Dahm \& Dimotakis 1987; Ferré et al. 1990; Mungal, Karasso \& Lozano 1991; Dimotakis 2000). However, in a recent paper Westerweel et al. (2005) showed that for a turbulent jet flow the engulfment motions do not dominate the entrainment process, because they only contribute a small percentage of the total jet mass flux. This result was in agreement with earlier numerical studies of a time-evolving jet by Mathew \& Basu (2002). The experimental findings indicated a jump in the mean axial momentum relative to the instantaneous jet interface, in correspondence to findings from numerical simulations for the turbulent/non-turbulent interface of a turbulent wake (Bisset, Hunt \& Rogers 2002) and of a time-evolving plane jet (da Silva \& Pereira 2008). Earlier studies (e.g. Kovasznay, Kibens \& Blackwelder 1970) could not identify any sharp discontinuity or 'jump' $\Delta U$ in the large-scale velocity fluctuations $\left(\sim U_{0}\right)$, i.e. $\Delta U \ll U_{0}$.

However, measurement of the instantaneous scalar profiles at the edges of jets and boundary layers and in other shear flows have shown that where there are differences in mean concentration $C$ of a scalar inside and outside the shear layer a significant fraction occurs as a step change $\Delta C$ at the interface (e.g. Mungal \& Hollingsworth 1989; Yoda, Hesselink \& Mungal 1994). Furthermore, Westerweel et al. (2005) showed that small-scale motions are dominant near the interface. Other recent findings appear to support this finding. Holzner et al. $(2007,2008)$ found that small-scale motion is dominant at the turbulent/non-turbulent interface of an advancing turbulent flow 
region generated by an oscillating grid. Similar findings were obtained by da Silva $\&$ Pereira (2008) for the turbulent/non-turbulent interface in a simulation of a timeevolving plane jet. They also found that the turbulent flow region resembles isotropic turbulence, and they could not find any signs of the existence of large-scale coherent motions at the instantaneous location of the interface. Also, it is reported by L'vov et al. (2008) that the spreading rate of a plane jet (i.e. the entrainment rate) is due to the randomness of the vortices, in contrast to the description of large-scale organized motion in relation to the entrainment rate (Yoda et al. 1994). The thickness $\ell_{\mathrm{i}}$ of the bounding interface is found to be of the order of the Taylor microscale $\left(\sim L_{x} / R e^{1 / 2}\right)$. This would be consistent with the concept of an interface at which the velocity jump is of the order of $U_{0}$ (Hunt, Eames \& Westerweel 2006; Ruban \& Vonatsos 2008). In the absence of a strong mean shear the limiting thickness may reduce to the Kolmogorov microscale $\left(\sim L_{x} / R e^{3 / 4}\right)$ (Corrsin \& Kistler 1955), as has been observed by Holzner et al. (2007, 2008).

This study provides new statistical and computational models of the outer region of turbulent flow, based on the finding that these results can be explained in terms of local models for the velocity and scalar fields in relation to the interface. Using the statistics of the interface in laboratory coordinates leads to physical concepts and practical models for first- and second-order moments in the outer zones of many types of turbulent shear flows. The approach avoids the numerical and conceptual difficulties inherent in using O. Reynolds's Eulerian averaging methods for intermittent flows with high local gradients. For example, in many numerical models of free turbulent flows it is common to assume a finite eddy viscosity in the outer irrotational flow region (e.g. Cazalbou, Spalart \& Bradshaw 1994). This can be considered as unphysical, although using zero eddy viscosity leads to an underestimation of the spreading rate of jets and boundary layers. Often this effect is masked by the numerical diffusion as a result of using a coarse simulation grid. In a previous paper (Westerweel et al. 2005) it was shown that the concept of a convoluted turbulent/non-turbulent interface predicts a finite eddy viscosity in the outer flow domain and provides a sound physical basis of the common practice of using a 'background' eddy viscosity. In this paper we expand the theoretical aspects of this result and extend the approach to the eddy diffusivity for a free shear flow. Taking into consideration the magnitudes of the conditional jumps and conditional mean gradients in the adjustment layer, we explain the turbulent Schmidt number in terms of the velocity and scalar jumps and the gradients' conditional velocity and scalar concentration in the adjustment layer.

The theoretical basis of our investigation is explained in $\S 2$. The experimental configuration, measurements and analysis of the experimental data are described in $\S 3$. The results are discussed in $\S 4$, with a summary and some suggestions for future studies given in $\S 5$.

\section{Theoretical}

\subsection{Principal concepts}

The convoluted moving interface may be idealized as a plane surface, when the engulfing motions only take place over a small proportion of the interface (see figure 2). The analysis of the engulfing mechanism by Hunt, Eames \& Westerweel (2008) shows that this assumption of infrequent engulfment events is valid if their streamwise length scale is less than the width of the shear layer. This occurs in flows like jets or wakes with significant mean curvature; it is not valid for plane free shear layers. Consider a scalar quantity $\theta$ that defines the turbulent flow region, i.e. $\theta=0$ in 
the irrotational external flow region $\mathscr{E}$ and $\theta>0$ in the turbulent flow region $\mathscr{A}$. The interface between the turbulent and irrotational flow regions is defined by a jump $\Delta \theta$, with the interface moving at a velocity $E_{b}$ into the irrotational flow region. A simple control volume analysis shows that the jump $\Delta \theta$ at the interface obeys the following condition:

$$
E_{b} \Delta \theta=-F_{\theta},
$$

where $F_{\theta}$ is the flux of $\theta$ into the control volume. Equation (2.1) implies that for $E_{b} \neq 0$ there must exist a non-zero jump $\Delta \theta$ when $F_{\theta}>0$. Many previous authors (e.g. Reynolds 1972) do not distinguish between $E_{b}$ and the mean inward velocity $V$ (see Turner 1986).

The instantaneous velocity field $u_{i}^{*}$ can be expressed both in terms of the mean and fluctuating component in the laboratory coordinates $\left(\overline{U_{i}}, u_{i}\right)$ and in coordinates defined relative to the interface $\left(\left\langle U_{i}\right\rangle, \tilde{u}_{i}\right)$, so that

$$
u_{i}^{*}=\overline{U_{i}}+u_{i}(\boldsymbol{x})=\left\langle U_{i}\right\rangle+\tilde{u}_{i}\left(x, y-y_{i}, z, t\right) .
$$

For convenience we adopt the following abbreviated notation: $\langle U\rangle=\left\langle U_{i}\right\rangle$ and $\tilde{\tau}=$ $-\langle\tilde{u} \tilde{v}\rangle$. Note that jumps $\Delta U$ and $\Delta \tau$ occur in the conditional mean velocity $\langle U\rangle$ and the local Reynolds stress $\tilde{\tau}$, respectively, across the interface. When the local pressure gradient is zero, the local momentum balance near the interface yields

$$
E_{b} \Delta U \cong \Delta \tilde{\tau} .
$$

(Note that the interface velocity is not constant, so that (2.3) is approximate.) On the turbulent side of the interface, where $n / \ell_{\mathrm{i}} \gg 1$, the mean velocity relative to the interface has a gradient $\partial\langle U\rangle / \partial n$ (Kovasznay et al. 1970; Bisset et al. 2002). This shear distorts the turbulence so that the eddy viscosity has an approximately constant value $v_{e} \sim v^{2} T_{L}$, where $T_{L}$ has a value characteristic of the outer region of shear layers $\left(T_{L} \sim 2 /(\partial\langle U\rangle / \partial n)\right)$ (Townsend 1976, p. 244). Therefore

$$
\tilde{\tau}=\Delta \tilde{\tau} \cong-v_{e} \frac{\partial\langle U\rangle}{\partial n} .
$$

(Note that $n$ is positive, pointing from the interface inwards into the turbulent flow region.) Similar relations are valid between the jumps in the mean scalar (e.g. concentration) and scalar flux $F_{C}(=\overline{v c})$, i.e.

$$
E_{b} \Delta C_{I}=-\Delta F_{C}
$$

and

$$
\Delta F_{C}=-D_{e} \frac{\partial\langle C\rangle}{\partial n},
$$

where $D_{e}$ is the eddy diffusivity.

Let $U_{S}$ and $C_{S}$ be the magnitudes of the changes in $\langle U\rangle$ and $\langle C\rangle$ across the main turbulent shear layer, between $n=0$ and $n \sim h$ (see figure 2). Then from (2.4) and (2.6)

$$
\left(\frac{\Delta U}{U_{S}}\right) /\left(\frac{\Delta C}{C_{S}}\right) \cong \frac{v_{e}}{D_{e}},
$$

where $v_{e}$ and $D_{e}$ are the eddy viscosity and eddy diffusivity respectively. Note that $D_{e}$ is typically twice as great as $v_{e}$ in the outer part of the shear layers; this is an experimental result that can also be derived from rapid distortion theory (Townsend 1976, p. 359). This is why the magnitude of the scalar jump $\Delta C$ across the interface layer is significantly greater than that of the velocity jump $\Delta U$ for jets and wakes 
(and similar flows), relative to the changes in $\langle C\rangle$ and $\langle U\rangle$ across the shear layer (e.g. Bisset et al. 2002).

As indicated in $\S 3$, the spatial resolution of the scalar measurement in our experiment was insufficient to resolve all relevant scales down to the Batchelor scale. However, the limitations of finite spatial resolution have less influence on the mean values of the passive scalar. Therefore, (2.7) provides an alternative evaluation for the scalar and momentum transport at the turbulent/non-turbulent interface.

Note that using other techniques, Buch \& Dahm (1996) showed that it was possible to fully resolve both the fluid motion and the scalar fluctuations for a liquid flow with $S c \gg 1$.

\subsection{Dynamical analysis relative to the interface}

The statistical dynamics of the engulfing and nibbling processes (which determine $E_{b}$ ) can be analysed by considering the terms in the equation for the kinetic energy $\overline{q^{2}}\left(=(1 / 2) \overline{u_{i}^{2}}\right)$ and mean square vorticity $\overline{\omega^{2}}$ when they are expressed in a fixed frame and a frame moving with the interface.

In a fixed frame (ignoring streamwise and spanwise derivatives)

$$
\frac{\partial \overline{q^{2}}}{\partial t}+\bar{V} \frac{\partial \overline{q^{2}}}{\partial y}=P-T_{r}-\varepsilon
$$

with

$$
P \cong-\overline{u v} \frac{\partial \bar{U}}{\partial y}, \quad T \cong \frac{\partial}{\partial y}\left(\overline{v q^{2}}+\overline{v p}\right), \quad \varepsilon=v \overline{\left(\frac{\partial u_{i}}{\partial x_{j}}\right)^{2}},
$$

where $P, T$ and $\varepsilon$ are the production, (radial) turbulent transport and dissipation terms, respectively. Consider a frame moving with the interface at velocity $V_{\mathrm{i}}-E_{b}$, using the notation in figure $2(a)$; then

$$
E_{b} \frac{\partial\left\langle\tilde{q}^{2}\right\rangle}{\partial n}=P-\varepsilon-\frac{\partial}{\partial n}\left(\left\langle\left\langle\tilde{v} \tilde{q}^{2}\right\rangle+\langle\tilde{v} \tilde{p}\rangle\right) .\right.
$$

Integrating across the thin interface (see figure 2) shows that the jump in the advective energy flux as the interface moves at speed $E_{b}$ has to balance the integrated contribution of $P$ in the interface plus the jump in the diffusive energy flux. The energy dissipation rate is of order unity, and its integral is small (Holzner et al. 2007). Thus (with the direction of integration into the turbulent flow domain; see figure 2)

$$
\begin{aligned}
E_{b} & \cong-\frac{\langle\tilde{u} \tilde{v}\rangle \Delta U+\Delta\left(\left\langle\tilde{v} \tilde{q}^{2}\right\rangle+\langle\tilde{v} \tilde{p}\rangle\right)}{\Delta\left\langle\tilde{q}^{2}\right\rangle} \\
& \cong-\alpha_{s h} \Delta U-\alpha_{t u r b} \sqrt{\left\langle\tilde{v}^{2}\right\rangle} .
\end{aligned}
$$

This shows how the nibbling process depends on eddies generated by the interface shear layer plus eddy transport (which would dominate in the experiments without shear; see Holzner et al. 2007, 2008). The coefficient $\alpha_{\mathrm{sh}}=\langle\tilde{u} \tilde{v}\rangle / \Delta\left\langle\tilde{q}^{2}\right\rangle$ can be determined from the measured conditional flow statistics, but since $\langle\tilde{v} \tilde{p}\rangle$ cannot be determined in our measurements, we consider the enstrophy equation to estimate $\alpha_{\text {turb }}$.

The analysis of the equation for the enstrophy in interface coordinates leads to

$$
\left(V_{\mathrm{i}}+E_{b}\right) \frac{\partial\langle\widetilde{\omega}\rangle}{\partial n}=P_{\omega}^{(L)}+T_{\omega}^{(N L)}-\varepsilon_{\omega},
$$


where

$$
P_{\omega}^{(L)} \sim\left\langle\widetilde{\omega}^{2}\right\rangle \frac{\partial\langle U\rangle}{\partial n}
$$

is the linear vorticity production term and

$$
T_{\omega}^{(\mathrm{NL})} \sim\left\langle\widetilde{\omega}_{i} \widetilde{\omega}_{n} \frac{\partial u}{\partial n}\right\rangle \sim \frac{\partial}{\partial n}\left\langle\tilde{v} \widetilde{\omega}^{2}\right\rangle
$$

the nonlinear transport term. Since the mean square vorticity in the interfacial layer is primarily locally produced with local dissipation, it follows that

$$
E_{b} \sim-\frac{\left\langle\tilde{v} \widetilde{\omega}^{2}\right\rangle}{\left\langle\widetilde{\omega}^{2}\right\rangle} \cong-\alpha_{\text {turb }} \sqrt{\left\langle\tilde{v}^{2}\right\rangle} .
$$

Near the interface when $\tilde{v}>0$, i.e. velocity fluctuations are directed towards the turbulent region from the irrotational flow region outside the interface, $\tilde{\omega}^{2} \cong 0$, whereas for $\tilde{v}<0, \tilde{\omega}^{2}>0$. Therefore, assuming these events are equally likely, it follows that $\alpha_{\text {turb }}=0.5$ in (2.12). This is tested against the experimental results in $\S 4$.

\subsection{Dynamical analysis in laboratory coordinates}

By using the analysis of the previous section relative to the moving interface, together with the p.d.f. $p\left(y_{\mathrm{i}}\right)$ of the interface position, it is possible to derive the relations between the mean fluxes and mean gradients for the velocity and scalar concentration. These can be compared with the relations used in engineering and environmental calculations. Some of these assume the form of an eddy viscosity $v_{e}(y)$ and eddy diffusivity $D_{e}(y)$, while others postulate approximate equations for moments of the velocity field. However, the latter approach is sensitive to the numerical methods used at the outer edge at which the turbulence tends to zero (Cazalbou et al. 1994).

The fluxes and mean profiles near the moving interface frame can be locally approximated by a step function adjacent to a linear gradient (Bisset et al. 2002, §4). For the velocity flux and conditional mean vorticity

$$
\begin{aligned}
\tilde{\tau}(n) & =H(n) \Delta \tau, \\
\left\langle\Omega_{z}\right\rangle(n) & =\delta(n) \Delta U+H(n) \frac{\partial\langle U\rangle}{\partial n} .
\end{aligned}
$$

Here $\delta(s)$ and $H(s)$ are the Dirac $\delta$ function and Heaviside step function respectively.

To calculate the mean values of the Reynolds stress and the vorticity in a laboratory frame relative to the mean position $\overline{y_{\mathrm{i}}}$, we use the experimental result that the probaility density function (p.d.f.) of $y_{\mathrm{i}}$ is Gaussian with a standard deviation $\sigma_{\mathrm{i}}$, where $\sigma_{\mathrm{i}}$ is typically of order of the integral length scale (Townsend 1976). Convolution of the p.d.f. $p\left(y_{\mathrm{i}}\right)$ of the interface position with the expressions in (2.17) and (2.18) yields (Westerweel et al. 2005)

$$
\begin{gathered}
\bar{\tau} \cong \frac{\Delta \widetilde{\tau}_{\mathrm{i}}}{2}\left[1+\operatorname{erf}\left(\frac{\Delta y}{\sqrt{2} \sigma_{\mathrm{i}}}\right)\right] \cdot \beta, \\
-\Omega_{z} \cong \underbrace{\frac{1}{2} \frac{\partial\langle U\rangle}{\partial n}\left[1+\operatorname{erf}\left(\frac{\Delta y}{\sigma_{\mathrm{i}} \sqrt{2}}\right)\right] \cdot \beta}_{A(\Delta y)}+\underbrace{\frac{\Delta U}{\sigma_{\mathrm{i}} \sqrt{2 \pi}} \exp \left(-\frac{\Delta y^{2}}{2 \sigma_{\mathrm{i}}^{2}}\right) \cdot \beta}_{B(\Delta y)},
\end{gathered}
$$

where $\Delta y=y-\overline{y_{\mathrm{i}}}$ and $\beta$ is the fraction of jet fluid at the mean position of the interface. The factor $\beta$ accounts for the fact that part of the fluid within the jet 
envelope is engulfed irrotational fluid that does not contribute to the mean vorticity $\Omega_{z}$ (Westerweel et al. 2005; see $\S 4$ ). The result (2.20) shows that the contribution of a significant peak in the conditional vorticity makes a significant contribution to the mean vorticity observed in a laboratory reference frame (Westerweel et al. 2005). From (2.19) and (2.20), it follows that the eddy viscosity $v_{e}$, defined in laboratory coordinates, as the ratio $\bar{\tau} /(\partial \bar{U} / \partial y)\left(=v_{e}\right)$ decreases from its value $v_{e_{s}}$ in the adjustment region $(\mathscr{A})$, where the mean shear is approximately constant, to its value $v_{e_{i}}$ near the interface, i.e.

$$
v_{e}(\Delta y=0) \equiv v_{e_{\mathrm{i}}}=\frac{v_{e_{s}}}{1+\lambda},
$$

where

$$
\lambda=\frac{\Delta U /\left(\sqrt{2 \pi} \sigma_{\mathrm{i}}\right)}{\frac{1}{2}(\partial\langle U\rangle / \partial n)} \sim 1 .
$$

Well outside the interface, where $(-\Delta y) \gg \sigma_{\mathrm{i}}$,

$$
\nu_{e} \rightarrow \frac{v_{e_{s}}}{1+4 \sqrt{\pi} \lambda}
$$

This is consistent with many measurements and Prandtl's hypothesis (Prandtl 1956) that the eddy viscosity is finite at the outer edge of shear layers and eventually decreases to a smaller constant value outside the layer (Westerweel et al. 2005).

The assumption in (2.4) and (2.17) that $v_{e}(n)$ is constant in $(\mathscr{A})$ has some justification even though the large eddies are damped near the interfacial layer (e.g. Hunt $\&$ Durbin 1999; Strang \& Fernando 2001). This is because the straining of the impacting eddies leads to upscale transfer of energy, which is an important mechanism in maintaining the sharpness of the interface.

The mean velocity $U$ and mean concentration $C$ in the outer jet region in laboratory coordinates can also be derived from the conditional mean velocity $\langle U\rangle$ and conditional mean concentration $\langle C\rangle$ profiles near the interface (i.e. $n \ll 2 b_{u}$ ),

$$
\begin{aligned}
& \langle U\rangle \cong H(n) \Delta U+n \cdot H(n) \frac{\partial\langle U\rangle}{\partial n}, \\
& \langle C\rangle \cong H(n) \Delta C+n \cdot H(n) \frac{\partial\langle C\rangle}{\partial n} .
\end{aligned}
$$

Convolution of (2.24) with $p\left(y_{\mathrm{i}}\right)$ yields

$$
\begin{aligned}
U(\Delta y) \cong\left(\Delta U+\Delta y \cdot \frac{\partial\langle U\rangle}{\partial n}\right) \cdot & \frac{1}{2}\left[1+\operatorname{erf}\left(\frac{\Delta y}{\sigma_{\mathrm{i}} \sqrt{2}}\right)\right] \cdot \beta \\
& +\frac{\partial\langle U\rangle}{\partial n} \cdot \frac{\sigma_{\mathrm{i}}}{\sqrt{2 \pi}} \exp \left(-\frac{1}{2} \frac{\Delta y^{2}}{\sigma_{\mathrm{i}}^{2}}\right) \cdot \beta,
\end{aligned}
$$

where $\beta$ is the fraction of jet fluid within the jet envelope, following (2.20). A similar expression is found for the mean concentration $C$. These expressions are only valid in the outer region of the jet, i.e. $\left|y-y_{0}\right| / b_{u} \gg 1$. Given that $\partial\langle U\rangle / \partial n \sim U_{c} / 2 b_{u}$ and $\partial\langle C\rangle / \partial n \sim C_{c} / 2 b_{u}$, it is found that $\Delta C / C_{c}>\Delta U / U_{c}$ and that, in laboratory coordinates, the jet half-width $b_{c}$ for the scalar field is larger than the jet half-width $b_{u}$. This is tested in $\S 4$ for the experimental data. 


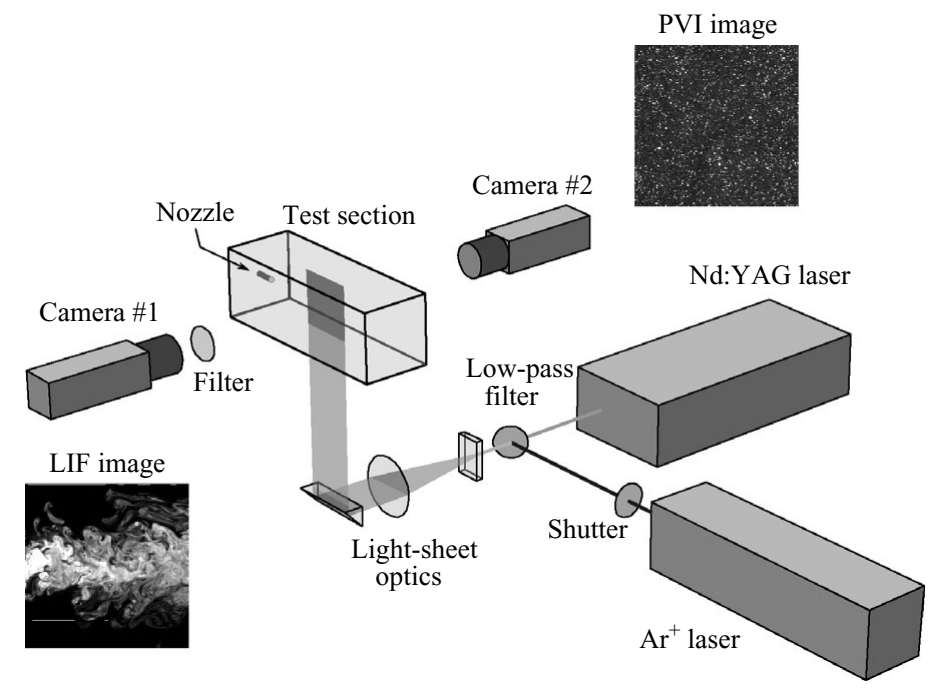

FIGURE 3. Schematic of the optical configuration for the combined PIV/LIF measurement, after Fukushima et al. (2002).

\section{Experiment}

The measurements were carried out with a combined particle image velocimetry/laser-induced fluorescence (PIV/LIF) measurement system. The original experiment was designed for the measurement of turbulent fluxes in a self-similar turbulent jet, for which the flow conditions match those of a direct numerical simulation (DNS) by Boersma, Brethouwer \& Nieuwstadt (1998). The details of the experimental configuration and of the comparison with the DNS results and other experimental data (Wygnanski \& Fiedler 1969; Panchapakesan \& Lumley 1993; Webster, Roberts \& Ra'ad 2001) are given in an earlier publication (Fukushima, Aanen \& Westerweel 2002); in this section we only provide a description of the facility that is relevant to the investigation of the jet interface. A schematic diagram is shown in figure 3. The method for the detection of the interface and a preliminary analysis of a subset of the total data set with a $32 \times 32$ pixel interrogation resolution are described in a separate paper (Westerweel et al. 2002), and first results on the momentum transport conditional to the position of the interface using data reinterrogated at $16 \times 16$ pixel spatial resolution were reported previously (Westerweel et al. 2005). In this paper the analysis of the experimental data is extended to include the conditional scalar transport.

\subsection{Experimental set-up}

The experimental set-up consists of a rectangular $110 \times 110 \times 300 \mathrm{~mm}^{3}$ test section. The fluid in the test section and the jet is water at room temperature, which has a kinematic viscosity of $1.0 \mathrm{~mm}^{2} \mathrm{~s}^{-1}$. The jet fluid enters the test section as a fully developed laminar pipe flow through an L-shaped needle with an inner diameter $d$ of $1 \mathrm{~mm}$. The length of the straight section of the needle is $100 \mathrm{~mm}$. The mean velocity in the tube is $2 \mathrm{~m} \mathrm{~s}^{-1}$, implying a jet Reynolds number $(R e)$ of $2 \times 10^{3}$. The jet fluid is marked with a fluorescent dye (disodium fluorescein) that has a Schmidt number (Sc) of $2 \times 10^{3}$, so that the molecular diffusion of the dye is negligible with respect to the turbulent mixing of the jet with the ambient fluid. This means that the boundary of the jet fluid does not become blurred by the effect of molecular diffusion. The 
fluid is seeded with $5 \mu \mathrm{m}$ tracer particles (Durcal). The outflow part of the section is connected to a $50 \mathrm{~mm}$ diameter pipe connected to a large outflow section (Fukushima et al. 2002). This largely avoids a recirculation inside the test section (although a very small backflow of less than 0.02 times the local centreline velocity was observed for $x / d>90)$.

A planar cross-section of the test section is illuminated with a $1 \mathrm{~mm}$ thick light sheet and consists of the light from two lasers. The first laser is a continuous wave $\mathrm{Ar}^{+}$ laser, and the second laser is a twin-cavity frequency-doubled pulsed $\mathrm{Nd}$ :YAG laser. The $\mathrm{Ar}^{+}$laser is used for the LIF measurements and the twin Nd:YAG laser for the PIV measurements. The $\mathrm{Ar}^{+}$laser is fitted with an ethalon so that it only emits the $488 \mathrm{~nm}$ (viz. blue) light, and an electro-optical shutter in the laser beam determines the duration of the laser exposure. The beams of the two lasers are combined along the same optical path by means of a dichroic mirror (see figure 3).

The PIV and LIF images are recorded by two identical digital cameras (Kodak MegaPlus ES 1.0) on opposite sides of the light sheet, with their optical axes perpendicular to the light-sheet plane (see figure 3). The cameras have a spatial resolution of $992 \times 1004$ pixels and a framing rate of $30 \mathrm{~Hz}$. The cameras are fitted with identical lenses with a $55 \mathrm{~mm}$ focal length (Nikon Micro Nikkor). The image magnification is 0.27 , and the field of view corresponds to a $45 \times 45 \mathrm{~mm}^{2}$ area. The misalignment between the cameras is less than 3 pixels, which corresponds to about $130 \mu \mathrm{m}$ in the object domain.

The first camera records the fluorescent light emitted by the dye. The fluorescent dye has an absorption curve with a maximum near the wavelength of the $488 \mathrm{~nm}$ (blue) light from the $\mathrm{Ar}^{+}$laser, and it emits (green) light with wavelengths between 500 and $545 \mathrm{~nm}$ and a maximum emission intensity near $514 \mathrm{~nm}$. An optical filter is placed in front of the camera that transmits the fluorescent light and rejects the $488 \mathrm{~nm}$ (blue) light that is scattered from the tracer particles, so that the recorded intensity only represents the fluorescent light. The duration of the $\mathrm{Ar}^{+}$laser exposure is $2.4 \mathrm{~ms}$, using the electro-optical shutter.

The second camera records the light that is scattered by the tracer particles. The camera is operated in dual-frame mode, so that the two laser exposures are recorded in separate frames. The time delay between the laser pulses is $1.2 \mathrm{~ms}$, which is chosen in correspondence to the so-called one-quarter rule for the in-plane displacement (Keane \& Adrian 1992) near the jet centreline. In order to avoid exposure of the LIF camera during the PIV recording, and vice versa, the LIF recording is taken with a small time delay directly following the PIV recording and electro-optical shutters fitted to the cameras to avoid unwanted exposure. In this way the $532 \mathrm{~nm}$ (green) $\mathrm{Nd}$ :YAG light scattered from the tracer particles is not recorded by the LIF camera. The total duration of a single combined PIV/LIF measurement is $5 \mathrm{~ms}$, which is negligible with respect to the Kolmogorov time scale $(20 \mathrm{~ms})$ of the turbulent flow (see Fukushima et al. 2002). Data are collected at a rate of 15 frames s$^{-1}$ in 12 runs of $4 \mathrm{~s}$ each; after each run the measurement section is rinsed.

The amount of light that is emitted by the fluorescent dye is directly proportional to the intensity of the illumination and the local concentration of the dye (Walker 1987). The recorded images are corrected for the inhomogeneous illumination due to the Gaussian profile of the $\mathrm{Ar}^{+}$laser (Fukushima et al. 2002).

The PIV frame pairs are interrogated with a two-pass interrogation procedure. In the first pass the image pairs are interrogated using $32 \times 32$ pixel interrogation regions and a $16 \times 16$ pixel spacing. The result from the first pass is first validated in order to remove any spurious data and then interpolated onto a grid with an 


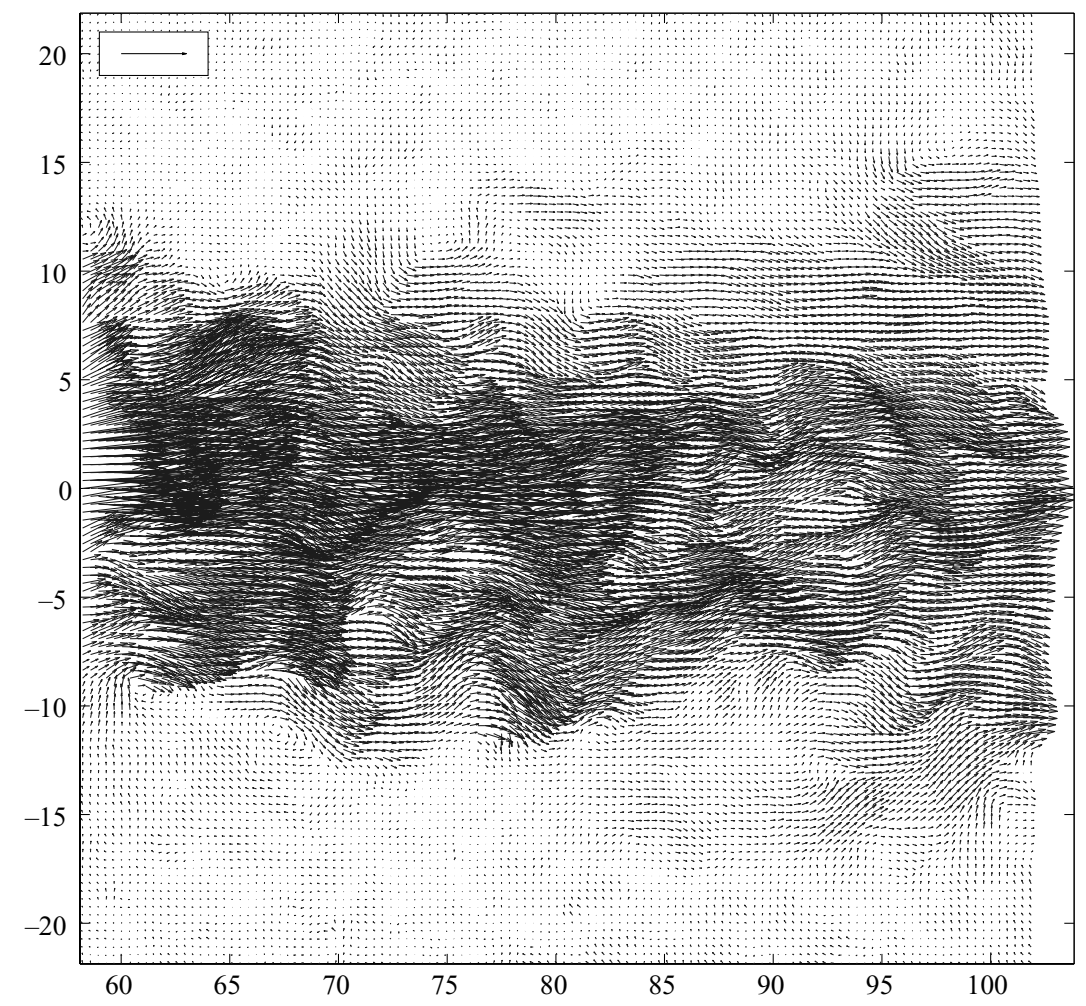

FIGURE 4. Example of the instantaneous velocity field. Dimensions are in mm relative to the nozzle location. The reference arrow in the box corresponds to a velocity of $250 \mathrm{~mm} \mathrm{~s}^{-1}$.

$8 \times 8$ pixel spacing. These data are used to determine the window offset for the second interrogation pass using $16 \times 16$ pixel interrogation regions. This procedure makes it possible to obtain reliable and accurate measurements while using very small interrogation domains (Westerweel, Dabiri \& Gharib 1997; Willert 1997). The fraction of spurious vectors in the final result is less than $2 \%$, which could be reliably detected by means of a median test (Westerweel 1994; Westerweel \& Scarano 2005) and were replaced by linear interpolation. The spurious vectors mainly occur in the central region of the jet, where there is a larger loss-of-correlation due to strong velocity gradients and large out-of-plane motion, whereas the jet boundaries contain significantly fewer spurious data.

Each PIV frame pair thus yields $(121 \times 121=) 14641$ velocity measurements at a spatial resolution of $0.37 \mathrm{~mm}$. The precision of the displacement measurements is estimated at $0.10-0.16$ pixels, which is better than $4 \mathrm{~mm} \mathrm{~s}^{-1}$ for the velocity. This is appreciably less than the estimated Kolmogorov velocity scale of $v \cong 12 \mathrm{~mm} \mathrm{~s}^{-1}$.

A typical example of the instantaneous velocity field obtained by PIV is shown in figure 4.

\subsection{Turbulent velocity and scalar statistics}

Combined PIV/LIF measurements were taken at three locations for (i) $20<x / d<60$, (ii) $60<x / d<100$ and (iii) $100<x / d<140$. The results for the mean centreline velocity $U_{c}$ and mean centreline concentration $C_{c}$ and for the jet half-widths $b_{u}$ and $b_{c}$ based on the velocity and concentration data respectively are reproduced in figure 5. As expected for a self-similar circular turbulent jet, $U_{c}$ and $C_{c}$ are inversely 
(a)

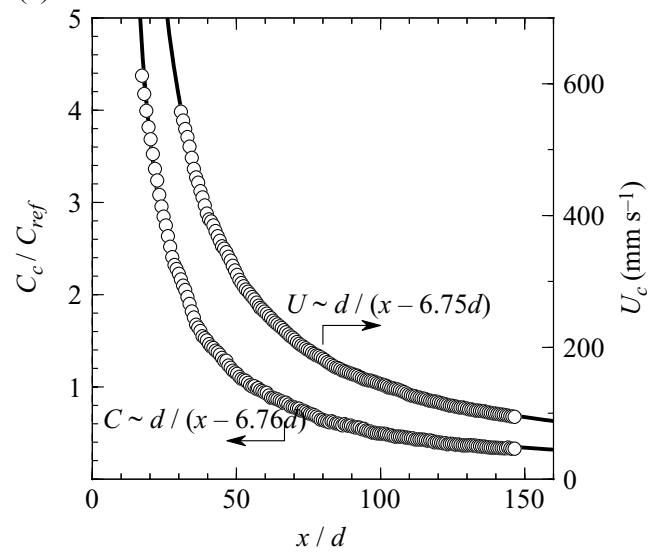

(b)

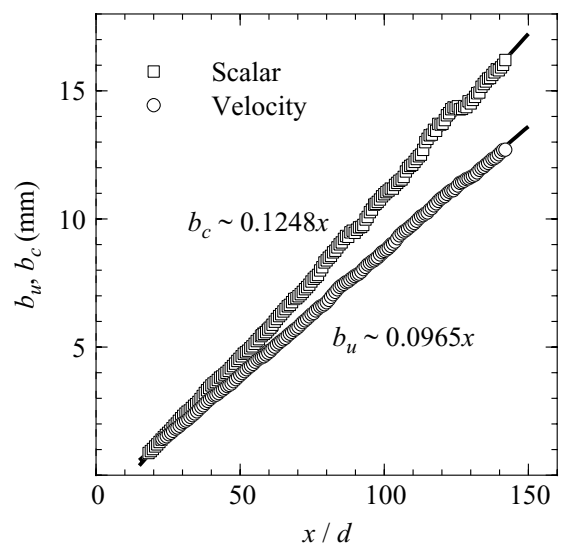

FIGURE 5. (a) The centreline mean velocity and mean concentration and $(b)$ the half-widths for the velocity field and the scalar field as functions of the distance from the nozzle.

(a)

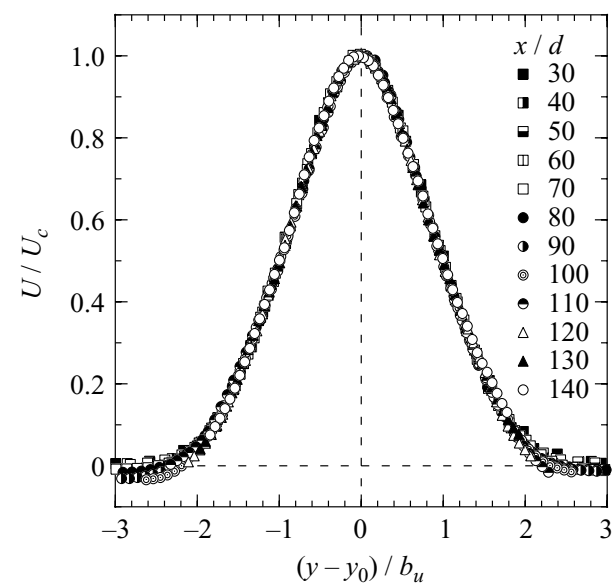

(b)

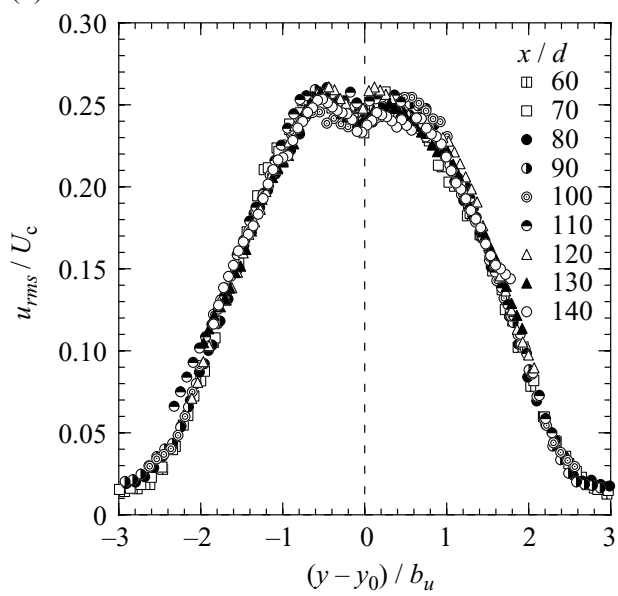

FIgURE 6. Profiles of the $(a)$ mean axial velocity and $(b)$ turbulent intensity of the axial velocity fluctuations at several distances from the jet nozzle.

proportional to the distance from the nozzle, whereas the jet half-widths $b_{u}$ and $b_{c}$ increase directly proportional to the distance from the nozzle. The jet half-width $b_{u}$ based on the velocity data is smaller than the jet half-width $b_{c}$ for the concentration data, with a ratio of $b_{u} / b_{c}=0.77$. This experimental finding is in agreement with results obtained by others (Hinze 1975; Chevray \& Tutu 1978; Chua \& Antonia 1990; Lubbers, Brethouwer \& Boersma 2001). The scaled radial profiles for the mean axial velocity and axial turbulence intensity for different distances from the jet nozzle are reproduced in figure 6 . The corresponding profiles for the concentration are very similar to those for the velocity (see Fukushima et al. 2002). The mean axial velocity profile appears to be self-similar for all results between $x / d=20$ and 140, although for the turbulence intensity self-similar profiles were only established for distances greater than about 50 nozzle diameters, but this may also be due to the limited spatial resolution of the PIV data. An extensive comparison of the present data with 
(a)

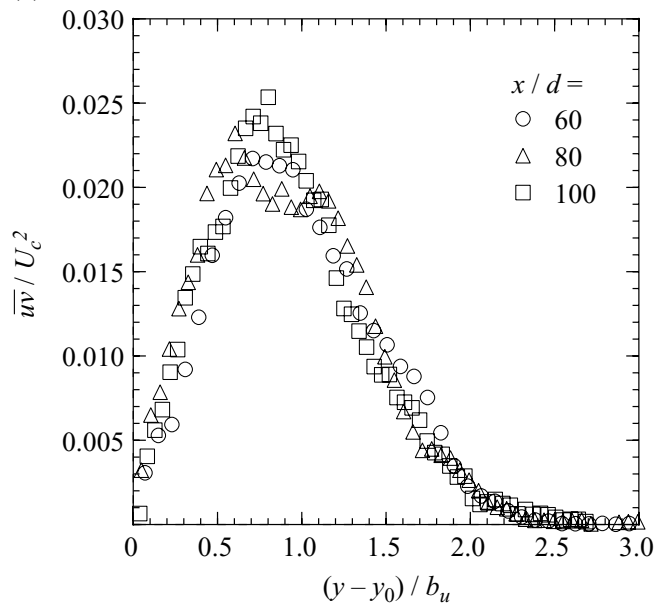

(b)

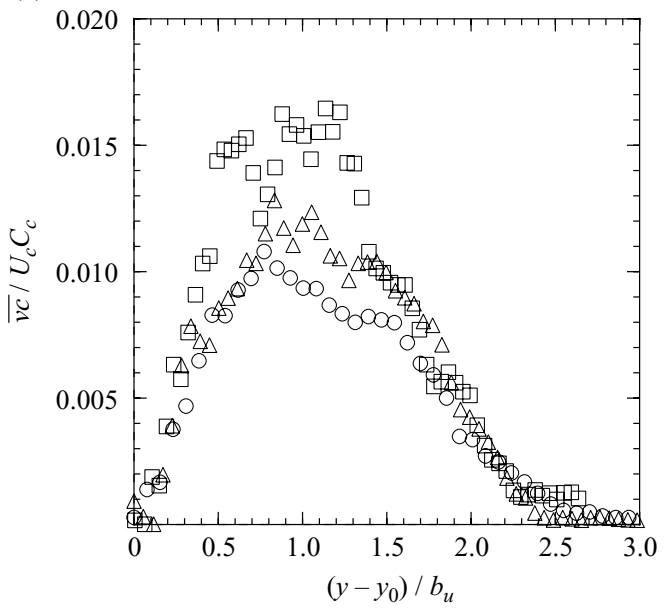

Figure 7. Profiles of the $(a)$ Reynolds stress and $(b)$ radial turbulent scalar flux at three distances from the jet nozzle.

various experimental data (Wygnanski \& Fiedler 1969; Panchapakesan \& Lumley 1993; Webster et al. 2001) and data from numerical simulations (Boersma et al. 1998; Lubbers et al. 2001) is provided in the paper by Fukushima et al. (2002).

Only the data from the second location for $60<x / d<100$ are used for the investigation of the turbulent/non-turbulent interface; the data from the third measurement location could not be used, since the jet boundary at this location was frequently not fully contained within the observation area.

The Kolmogorov length scale $\eta_{K}$ is determined from the estimated dissipation rate $\varepsilon$ and kinematic viscosity $v\left(\cong 1 \mathrm{~mm}^{2} \mathrm{~s}^{-1}\right)$. With $\varepsilon=0.015 U_{c}^{3} / b_{u}$ (Panchapakesan \& Lumley 1993) it is found that $\eta_{K}=0.08 \mathrm{~mm}$. Hence, the spatial resolution of the PIV data (conservatively estimated as the linear dimension of the interrogation domain, i.e. $0.37 \mathrm{~mm}$ ) is 4.6 times the Kolmogorov scale. This is within the range of $4-5$ times the Kolmogorov scale for which the velocity data can be considered as fully resolved, i.e. adequately resolving the turbulence statistics and instantaneous velocity derivatives (Wallace \& Foss 1995; Tropea, Yarin \& Foss 2007). The Taylor microscale $\lambda_{T}$ is then estimated from $\varepsilon \cong 15 \overline{u^{\prime 2}} / \lambda_{T}^{2}$ (Hinze 1975). Using $\left(\overline{u^{\prime 2}}\right)^{1 / 2} \cong 0.25 U_{c}$ (see figure $6 b$ ), it is found that $\lambda_{T} \cong 0.18 b$, which is almost twice the equivalent dimension of the interrogation domain (about $0.1 b_{u}$ ). Results for the two-point spatial correlation for the streamwise and transverse velocity fluctuations along $\left|y-y_{0}\right| / b_{u}(x)=0$ and 1 are reported in a paper by Ewing et al. (2007).

Figure 7 shows the Reynolds stress $\overline{u v}$ and radial scalar flux $\overline{v c}$ at three different positions. The scalar flux $\overline{v c}$ increases with downstream distance, as the fraction of unresolved scales decreases with downstream distance. An estimate of the spatial resolution of the LIF measurements was obtained by considering the spectral density of the grey values along the jet centreline over all 657 frames, corrected for inhomogeneities by first subtracting and then dividing the instantaneous grey values with the local mean grey value. From this we estimated the white noise level in the LIF images and determined an effective spatial resolution of $0.14 \mathrm{~mm}$. Given a Schmidt number $S c=2 \times 10^{3}$ and a Kolmogorov length scale $\eta_{K} \cong 80 \mu \mathrm{m}$, the estimated Batchelor scale is less than $2 \mu \mathrm{m}$, and evidently the scalar fluctuations are significantly under-resolved. We therefore use an alternative approach, described in 
$\S 2$, to relate the interface concentration jump, the conditional concentration flux and the entrainment velocity $E_{b}$.

\subsection{Detection of the interface}

In the present study we use a passive marker to mark the jet fluid and to determine the turbulent/non-turbulent interface. Ideally, the interface between the turbulent (viz. rotational) flow region and the non-turbulent flow region is determined directly from the vorticity. This is easily achieved in numerical studies (e.g. Bisset et al. 2002), but this is more complicated in experimental studies, as it requires the availability of spatially resolved three-component volumetric velocity data. This was achieved by Holzner et al. $(2007,2008)$ but only for the case of an advancing turbulent flow generated by an oscillating grid, which has no mean shear. For turbulent shear flows, such as jets, wakes and mixing layers, other methods have been employed. Kibens, Kovasznay \& Oswald (1974) developed a turbulence indicator for a hot-wire probe. A different approach is to use a passive scalar that is advected by the turbulent fluid motion. In many previous studies the temperature of the turbulent fluid is slightly raised, so that the measured temperature can be used as a turbulence indicator (Antonia 1981). A complication is the finite thermal diffusivity in gas flows, characterized by the Prandtl number $(\mathrm{Pr})$, which is about 0.7 for air at room temperature. In the present measurement we apply a fluorescent dye as a passive scalar, which has a very small diffusivity corresponding to a Schmidt number $(S c)$ of $2 \times 10^{3}$. Hence, effects of finite diffusivity of the dye are much less of a problem in comparison to previous studies using temperature as a passive scalar. This is apparent from estimating the characteristic diffusion time taking into consideration the experimental conditions. The characteristic time $t$ for the dye to diffuse over a length $\ell_{D}=\sqrt{2 D t}$ equal to the spatial resolution of the measurement $(0.37 \mathrm{~mm})$ is estimated to be $160 \mathrm{~s}$, which is a few orders of magnitude larger than the mean time between the emission from the jet nozzle and the passage through the measurement domain (estimated at 2-3s). Hence, the finite diffusivity of the dye can be ignored for the purposes of the present measurements.

For the detection of the interface, the LIF data are transformed into binary images by means of threshold detection: pixels with a grey value above the threshold value are labelled ' 1 ', i.e. jet fluid, and all other pixels are labelled ' 0 ', i.e. ambient fluid (see figure $8 a$ ). The threshold value is chosen in accordance with the procedure described by Prasad \& Sreenivasan (1989; see also Westerweel et al. 2002). Occasional patches of dye are seen that appear to be disconnected from the main jet body. This is the result of vortical eddies that break away from the interface, indicated as 'detrainment'. In the absence of a mean shear, the turbulence quickly decays (i.e. the vorticity vanishes by means of diffusion), while the dye remains and no longer indicates a turbulent flow region. For jets and wakes and free shear layers with no external fluctuations, detrainment is a small effect, because such patches are usually re-entrained within a few eddy time scales (Hussain \& Clark 1981). Fortunately, these patches occur disconnected to the main jet body, and we can simply exclude these regions from the analysis by removing them (figure $8 b$ ). In addition, from our PIV measurements we can verify that these detrained patches do not carry any significant vorticity. The turbulent jet interface is obtained from the object boundary as a continuous curve (figure $8 c$ ). The shape of the interface can be quite complex; i.e. in some places a vertical line can intersect the interface more than once. This particularly occurs whenever ambient fluid is being engulfed by the jet fluid. It is common to consider an envelope that defines the confinement of the turbulent fluid, including the patches 

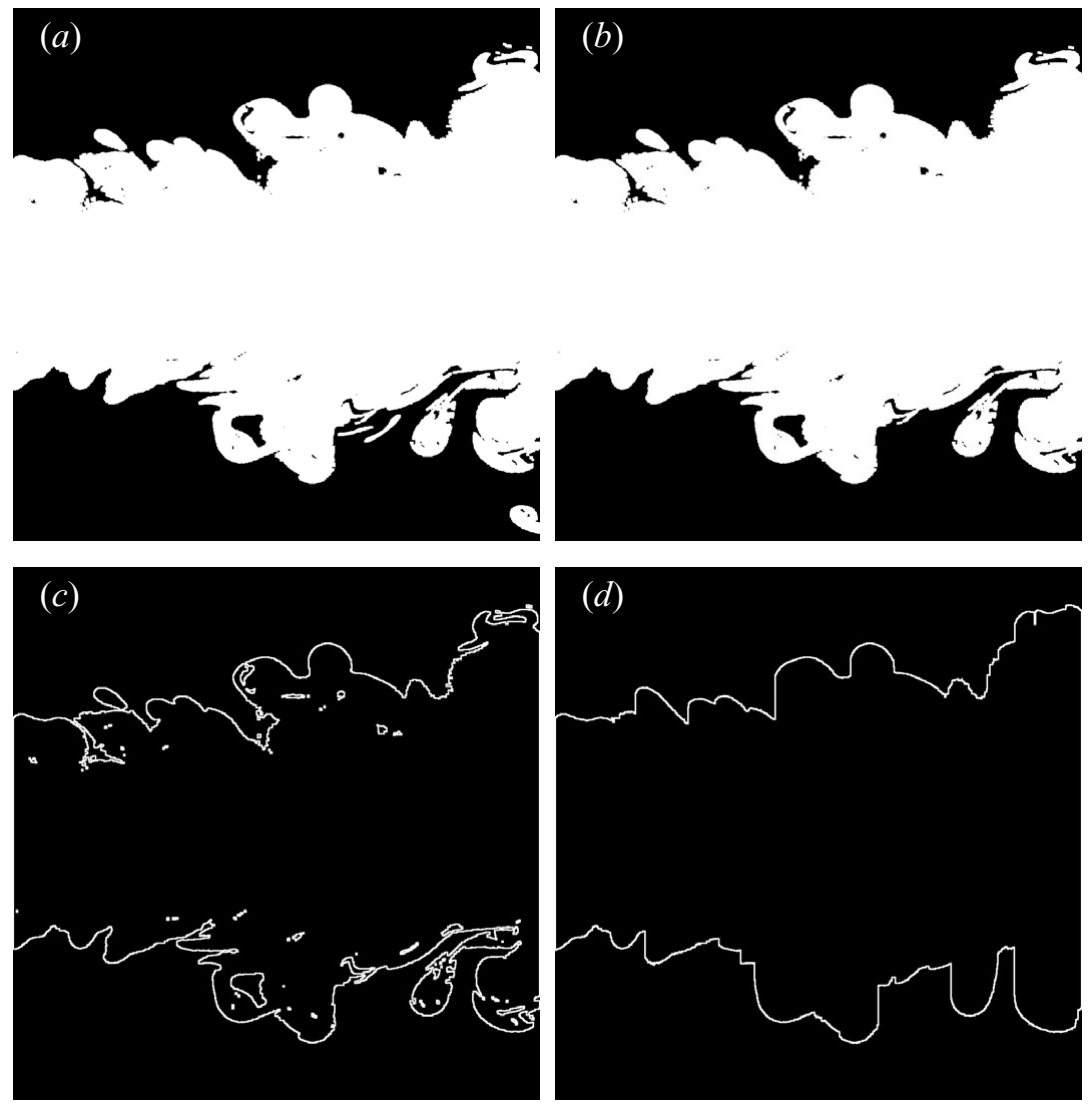

FiguRE 8. The detection of the interface and envelope. ( $a$ ) LIF image after thresholding; $(b)$ removal of disconnected objects; $(c)$ detected interface; and $(d)$ the interface envelope resulting from saving the outermost points in (c). See also Westerweel et al. (2002).

of engulfed irrotational fluid. Following Hernan \& Jimenez (1982) we determine this envelope by saving the outermost points of the interface along each vertical line (see figure $8 d$ ).

Sandham et al. (1988) used a similar approach by considering two contour levels of a diffusive scalar $(S c=0.25$ and 1.0) to define the boundary of the turbulent flow region in a DNS of a mixing layer. They determined the fraction of engulfed and mixed fluid within the envelope defined by the concentration contours. The same approach was used by Mathew \& Basu (2002), who applied it to a time-evolving jet.

The result for the envelope is quite insensitive to the value of the threshold. Essentially the same envelope would be detected when the threshold value is increased by 2 and even 3 times the optimal threshold value (see figure 9). The robustness of the interface detection is primarily owing to the fact that the scalar concentration has a large jump at the interface that is about 10 times the threshold value. However, one can observe that in the interior of the jet the fraction of grey regions (marking 'unmixed' fluid) increases for increasing detection threshold. Using a threshold that is much higher than the optimal value defined by Prasad \& Sreenivasan (1989) leads to a result that appears to show that the interior of the jet contains a significant fraction of unmixed fluid. Also, certain flow visualization studies with high contrast photography could lead to such a misleading impression. There are of course thin 

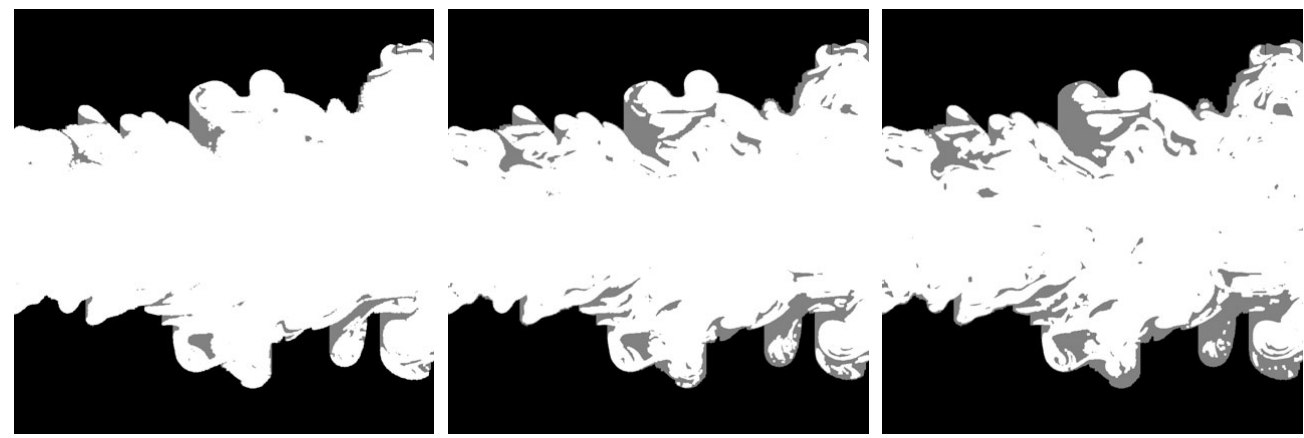

(a)

(b)

(c)

FIGURE 9. The result for the detected jet fluid for $(a) 1$, (b) 2 and (c) 3 times the threshold value determined by means of the method of Prasad \& Sreenivasan (1989). The grey area represents the fluid within the envelope shown in figure $8(d)$.

elongated layers even in the interior where the concentration is less than the average value, but the fluid is already partially mixed as is evident from the fact that the concentration has a significant non-zero value. This contrasts with the case of a free shear layer in which there is a significant level of unmixed fluid in the interior (Brown \& Roshko 1974; Broadwell \& Breidenthal 1982).

\subsection{Conditional sampling}

The flow properties are determined relative to the turbulent/non-turbulent interface by taking conditional statistics by averaging data at fixed distances relative to the jet envelope. This procedure is illustrated in figure 10, which shows the vorticity field of the instantaneous velocity field in figure 4 . The envelope from figure $8(d)$ has been superimposed, and the crosses indicate data points at a fixed distance from the envelope over which the data are averaged. This conditional averaging approach is described in detail by Bisset et al. (2002).

The velocity data are interpolated on a grid with a $1 \times 1$ pixel spacing to match the resolution of the LIF data, and the conditional statistics are determined for the axial and radial velocity components, Reynolds stress, vorticity and scalar concentration over a range of -300 pixels to +300 pixels on either side of the boundary.

\section{Results and discussion}

\subsection{Mean and turbulent profiles}

The p.d.f. of the measured envelope position $y_{\mathrm{i}}$ scaled with the jet half-width $b_{u}$ is plotted in figure 11. When the p.d.f. for $y_{\mathrm{i}}$ is compared against a normal distribution (represented by the dash-dotted curve), it appears that the p.d.f. is slightly skewed towards the outer region of the jet. The mean position of the envelope relative to the jet centreline is at $1.99 b_{u}$, and the most likely position is at $1.93 b_{u}$, which differ by only $3 \%$. The skew may possibly originate from the fact that the envelope represents the outermost position of the interface rather than the interface itself. However, the skew is very small and does not affect the further analysis. The width $\sigma_{\mathrm{i}}$ of the p.d.f. for $y_{\mathrm{i}}$ (defined as the standard deviation of the p.d.f.) is equal to $0.41 b_{u}$.

The turbulent flow statistics are taken conditionally with respect to the distance from the jet interface. Figure 10 shows the instantaneous jet interface superimposed on the instantaneous (out-of-plane component of the) vorticity $\omega_{z}$. (For brevity the 


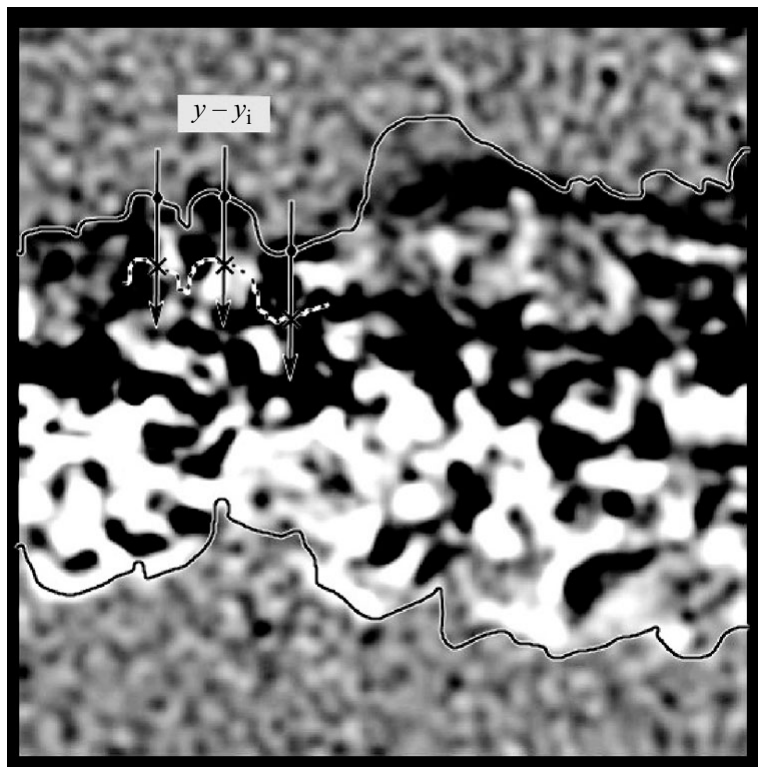

FIGURE 10 . The vorticity $\Omega_{z}$ and the jet envelope (continuous line). Conditional statistics are determined by averaging data at fixed distances relative to the envelope (indicated by the crosses and dashed line); see also Bisset et al. (2002).

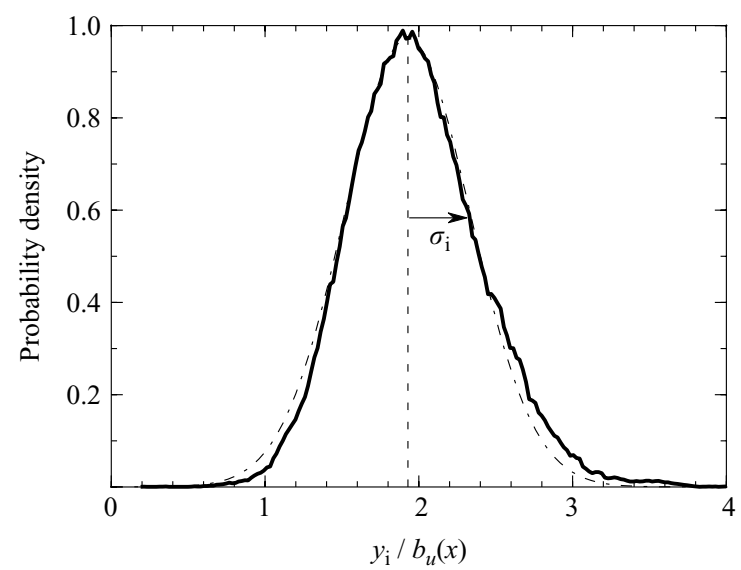

FIGURE 11. The p.d.f. of the interface position $y_{\mathrm{i}}$. The dash-dotted curve represents a normal distribution with a standard deviation $\sigma_{\mathrm{i}}$.

'out-of-plane component of the vorticity' will simply be referred to as 'vorticity'.) The conditional mean vorticity profile is plotted in figure 12 for three distances from the nozzle. The result clearly shows a jump in the value of the mean conditional vorticity and a nearly constant value of the vorticity on the inside of the interface. When the same data are plotted in a scaled fashion, i.e. the distance from the interface is scaled with the jet half-width $b_{u}$ and the vorticity with the half-width $b_{u}$ divided by the mean centreline velocity $U_{c}$, then all three profiles appear to be self-similar. The scaled constant value for the mean vorticity at the inside of the jet is about $0.55 U_{c} / b_{u}$. The result that the mean conditional vorticity vanishes on the irrotational side of the 

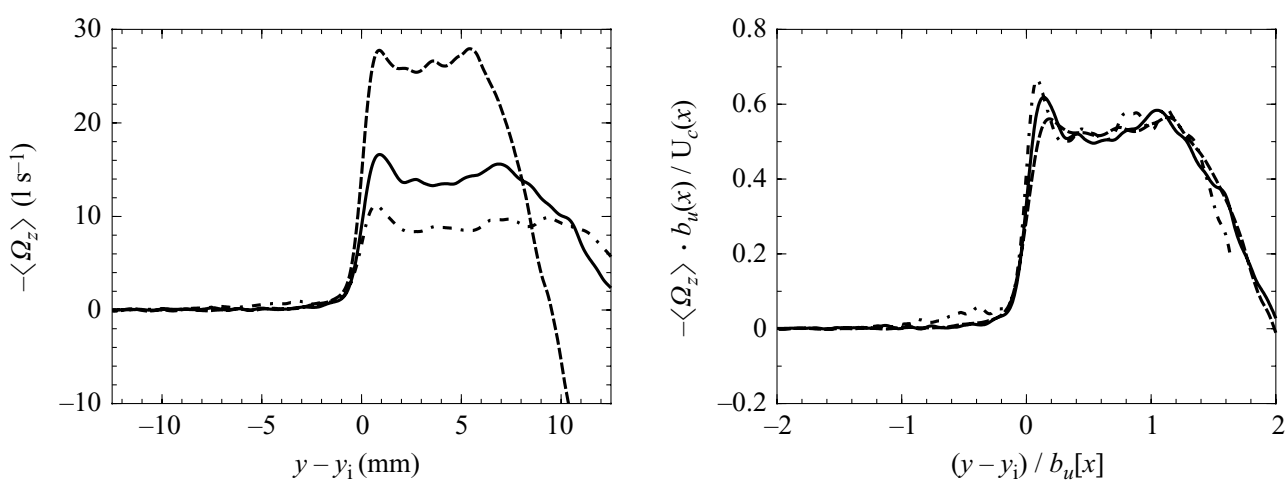

FIGURE 12. The mean conditional vorticity $\left\langle\omega_{z}\right\rangle$ as a function of the distance from the interface at three different distances from the nozzle: $x / d=49.3(---), 67.5(-)$ and $85.7(-\cdot-)$.

interface and shows a strong jump at the interface validates our approach for the detection of the interface.

In all three profiles for $\left\langle\Omega_{z}\right\rangle$ there appears a small peak at the inside of the interface. This peak demonstrates the tendency of a vortex sheet to form at the outer edge of the jet with a strength of order $U_{c}$. The peak in the mean conditional vorticity may alternatively be associated with small individual vortices with their axes normal to the plane of observation. Note that the vorticity in the thin shear layer does not scale with $U_{c} / b_{u}$. The width of the thin shear layer could be less than the finite dimensions of the interrogation domain; in that case the observed peak width and circulation would be determined by the (constant) dimensions of the interrogation domain. In order to validate this, additional measurements would be required, preferably in the region $x / d>100$ in which the relevant length scales would exceed the finite dimensions of the interrogation domain.

The graphs for the results for the conditional turbulence statistics are plotted in figures 13 and 14. The markers in these graphs indicate the nominal 8 pixel spacing between interrogation positions. The mean axial velocity $\langle U\rangle$ is essentially zero on the irrotational side of the interface and increases linearly with the distance from the interface in the turbulent region. The peak in $\left\langle\Omega_{z}\right\rangle$ suggests the existence of a small jump in $\langle U\rangle$ but can scarcely be resolved. Further analysis of $\langle U\rangle$ is described in $\S 4.2$. The mean radial velocity $\langle V\rangle$ is positive on the irrotational side of the interface; i.e. there is a net mass flow into the turbulent flow region. On the turbulent side of the interface $\langle V\rangle$ decreases and eventually becomes negative, which is in accordance with the gradual decrease of the mean axial velocity and an increase of the jet crosssection with downstream distance. The profile for the mean conditional concentration $\langle C\rangle$ in figure 13 shows a clear jump at the interface. The magnitude of the jump is about $0.3 C_{c}$. This explains why the detection of the interface (and jet envelope) is relatively invariant with respect to the choice of the threshold value (see $\S 3$ ). The mean conditional concentration also shows a nearly linear increase with respect to the distance from the interface in the turbulent flow region, but there is a region about $0.2-0.3 b_{u}$ wide near the interface in which the gradient of $\langle C\rangle$ nearly vanishes. This is indicative of a local mixing region that can be associated with the interface (Turner 1986, figure 9). The width of this mixing layer is of the order of the Taylor microscale. If a straight line is fitted to the profile for $\langle C\rangle$ and extrapolated to the 

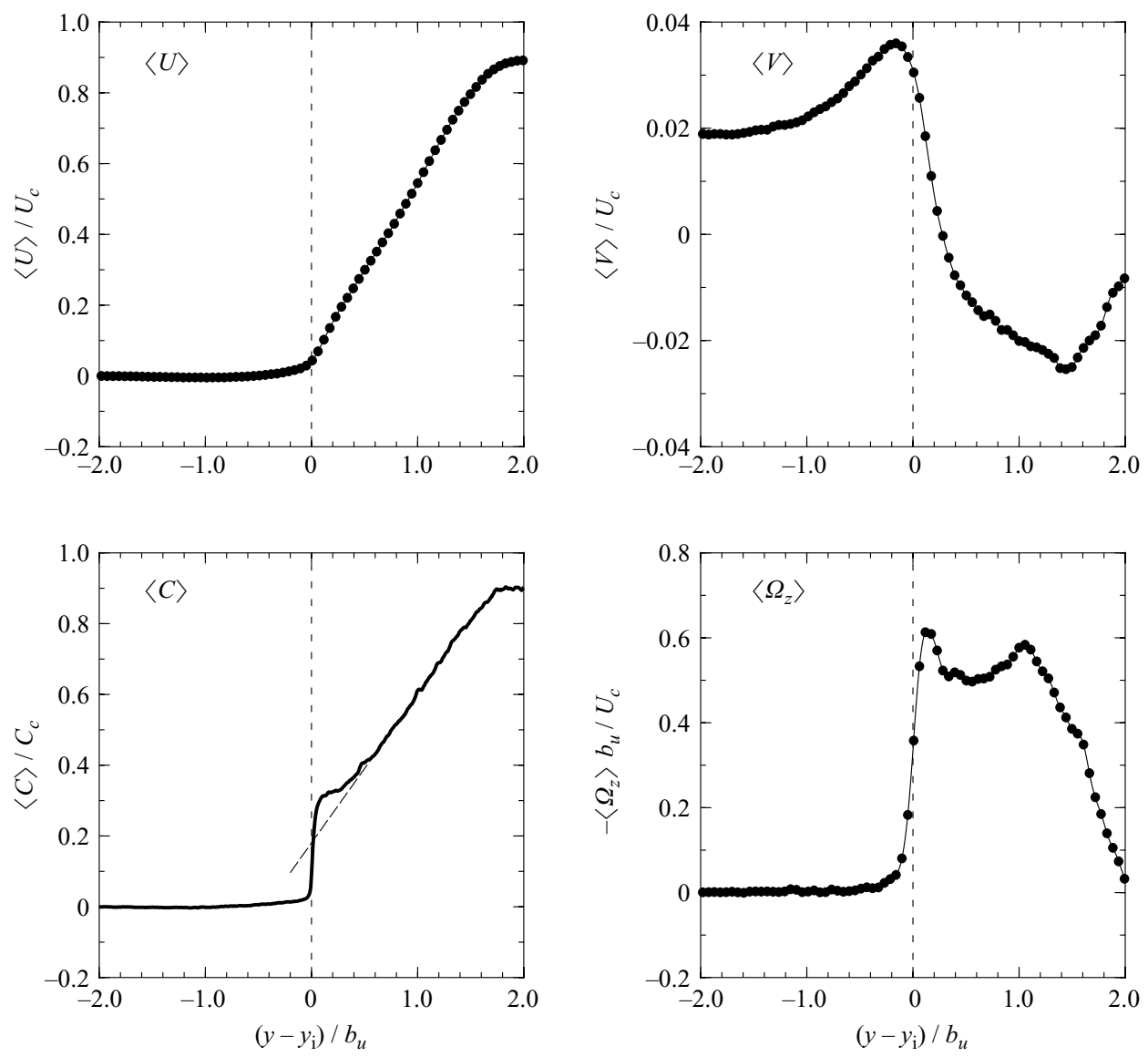

FIGURE 13. The conditional statistics.

position of the interface (see figure 13), then a value for $\Delta C$ equal to about $0.18 C_{c}$ is found.

The results in figure 14 for the root mean square (r.m.s.) conditional axial and radial velocity fluctuations, $\left\langle u^{2}\right\rangle^{1 / 2}$ and $\left\langle v^{2}\right\rangle^{1 / 2}$ respectively, show that velocity fluctuations exist on both sides of the interface. The profiles have a small jump at the interface of about $0.08 U_{c}$. The analysis in $\S 2.2$ shows that the entrainment velocity $E_{b}$ is proportional to $\Delta U$ and $\left\langle v^{2}\right\rangle^{1 / 2}$; this is discussed in further detail in $\S 4.4$. Figure 14 also shows the conditional Reynolds stress $\langle u v\rangle$. The turbulent momentum flux towards the interface is indicated in the graph. It is interesting to note that the conditional Reynolds stress $\langle u v\rangle$ has a small non-zero value at the irrotational side of the interface that does not contribute to the turbulent momentum transport (Pope 2000). This was also observed by Bisset et al. (2002).

Finally, figure 14 includes a graph of the profile of the scaled conditional vorticity fluctuations $\left\langle\omega_{z}^{2}\right\rangle^{1 / 2}$. Although an irrotational flow is expected on the outside of the jet interface, the experimental result yields non-zero values indicated by the dashdotted line. In the case of experimental PIV data, even for an irrotational flow finite values for $\left\langle\omega_{z}^{2}\right\rangle^{1 / 2}$ are found as a result of the finite measurement error in the displacement (Adrian 1991; Westerweel 2000). For the experimental parameters in the measurements, an r.m.s. error of less than 0.2 pixel is expected for the displacement 

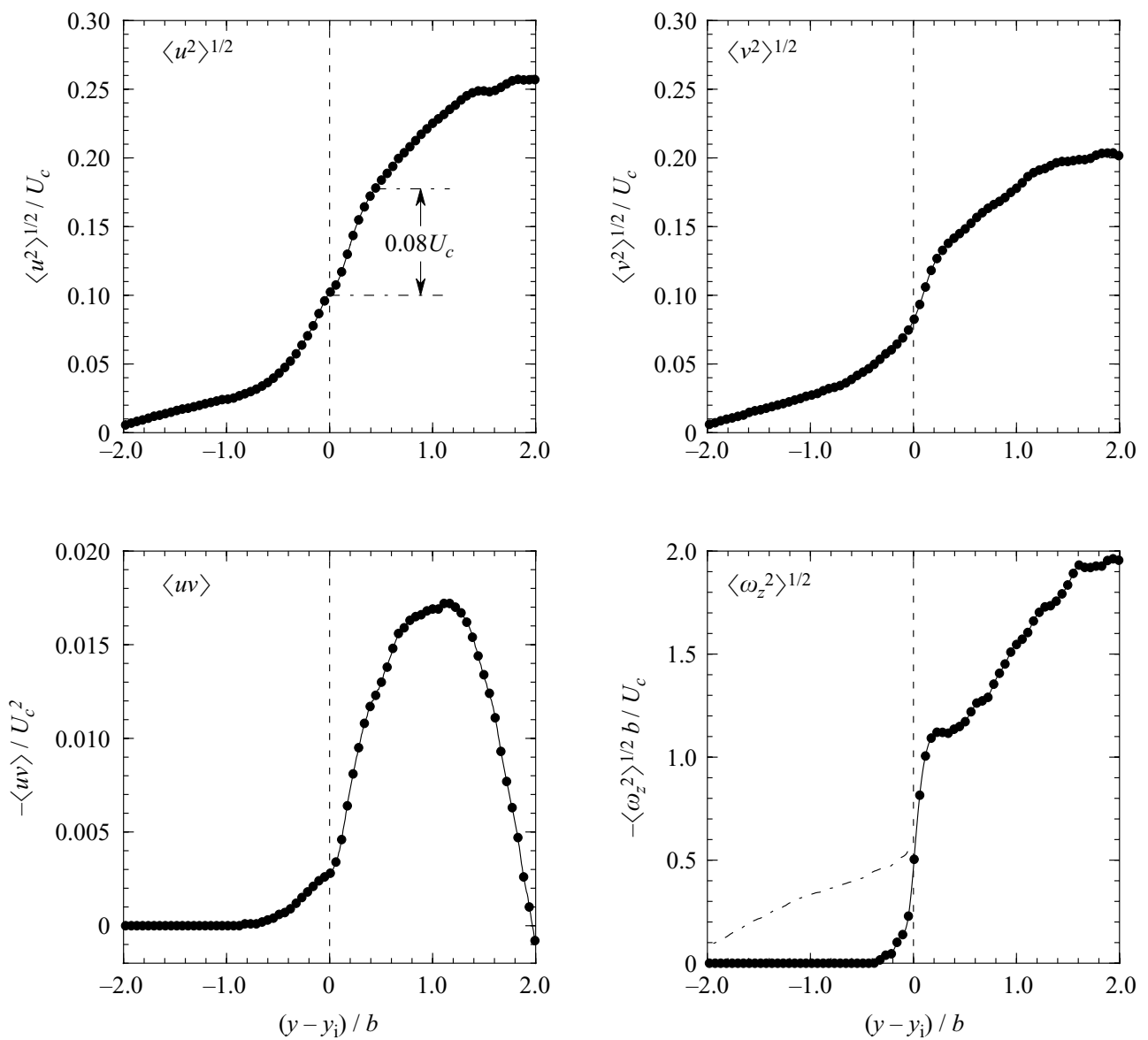

FIGURE 14. The conditional statistics (continued).

(Westerweel 2000; Stanislas et al. 2005). Given the numerical scheme for the estimation of the vorticity from the displacement data (Landreth \& Adrian 1990; Raffel, Willert \& Kompenhans 1998), the estimated contribution of the PIV measurement noise is subtracted from $\left\langle\omega_{z}^{2}\right\rangle^{1 / 2}$. The solid line in the graph for $\left\langle\omega_{z}^{2}\right\rangle^{1 / 2}$ in figure 13 shows the corrected data. The residual data on the irrotational side of the interface effectively vanish, whereas the correction is marginal on the turbulent side of the interface.

\subsection{Momentum jump at the interface}

Figure 15 contains an enlargement of the profile for $\langle U\rangle$ in figure 13. These are compared with two model profiles representing linear velocity profiles with and without an interface velocity jump that have been convoluted with a triangular kernel that represents the finite dimension of the PIV interrogation domain (figure 15 inset). The actual jump may also be smoothed by viscous effects. Figure 15 shows that the magnitude of the jump can be inferred from the deviation of the conditional velocity profile away from the interface. Also, it shows that the presence of a velocity jump is indicated by an inflection point in the measured conditional profile at the location of the interface. The measured result for $\langle U\rangle$ clearly features an inflection point and is very similar to the expected result for the convoluted discontinuous curve; this is consistent with presence of a jump in the result for $\langle U\rangle$. An extrapolation 


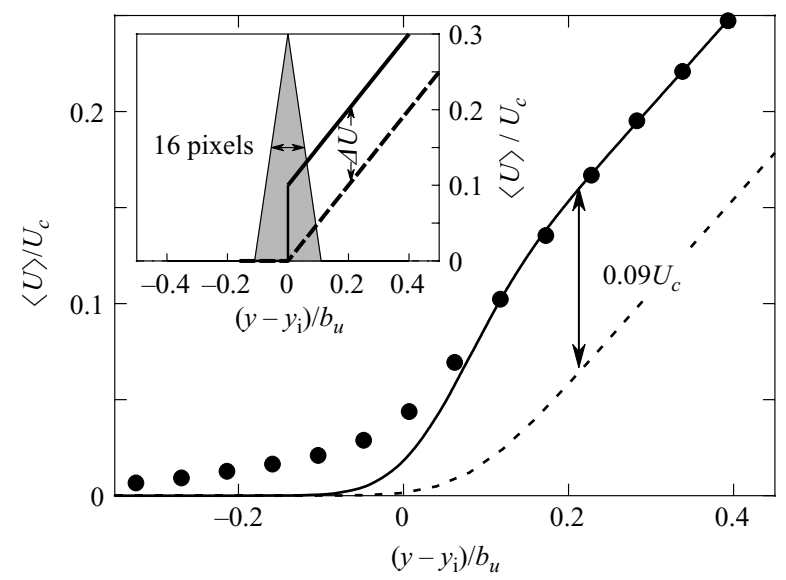

FIGURE 15. Detail of the conditional axial velocity near the interface. The solid and dashed lines are obtained by convolution of a triangular kernel (representing the finite $16 \times 16$ pixel dimension of the PIV interrogation domain) with linear profiles with and without a velocity jump $\Delta U$ at the interface (inset).

of the measured linearly increasing profile for $\langle U\rangle$ to the interface position shows that the jump $\Delta U$ is at least greater than $0.06 U_{c}$. Given the measured profile for $\langle U\rangle$ and taking into account the effect of the finite spatial resolution of the PIV data, it is estimated that the jump in $\langle U\rangle$ is $0.09 U_{c}$ (Westerweel et al. 2005). In order to determine the interface propagation velocity we need to determine the value for the conditional Reynolds stress $\langle u v\rangle$ at the interface, which is estimated at $0.007 U_{c}^{2}$. From the local analysis in $\S 2$, this implies a propagation velocity of the interface, or boundary entrainment velocity, of $E_{b}=-0.007 U_{c}^{2} / 0.09 U_{c}=-0.07 U_{c}$. According to Turner (1986) the outward boundary entrainment velocity $E_{b}$ is given by

$$
E_{b}=-2 V,
$$

where $V$ is the mean inward radial velocity at the jet interface. From the graph for $\langle V\rangle$ in figure 13 we obtain $\langle V\rangle=0.033 U_{c}$, so that $E_{b}=-0.067 U_{c}$. This theoretical value for the propagation velocity $E_{b}$ is in close agreement with the measured value that is obtained from the measured jump $\Delta U$ in the axial velocity and the measured turbulent momentum flux $\langle u v\rangle$.

The results in figures 13 and 14 show a strong resemblance to the results obtained by Bisset et al. $(1998,2002)$ from DNS data of a turbulent planar wake. The wake data also exhibit a peak in $\left\langle\Omega_{z}\right\rangle$ at the location of the interface.

\subsection{Mass flux}

The jet envelope, defined in figure $8(d)$, describes the outer boundary of the jet. The actual interface is quite contorted, so that for a finite scale of resolution it is possible to find irrotational fluid within the detected jet envelope. This is shown in figure $9(a)$, where turbulent jet fluid is shown in white, and irrotational fluid within the envelope is shown in grey. The number of grey pixels would be a measure of the amount of engulfed irrotational fluid that is entrained by the jet, whereas the total number of pixels within the jet envelope defining an enclosed volume (or mass) within the jet boundary would be proportional to the total jet mass (Sandham et al. 1988; Mathew \& Basu 2002). 


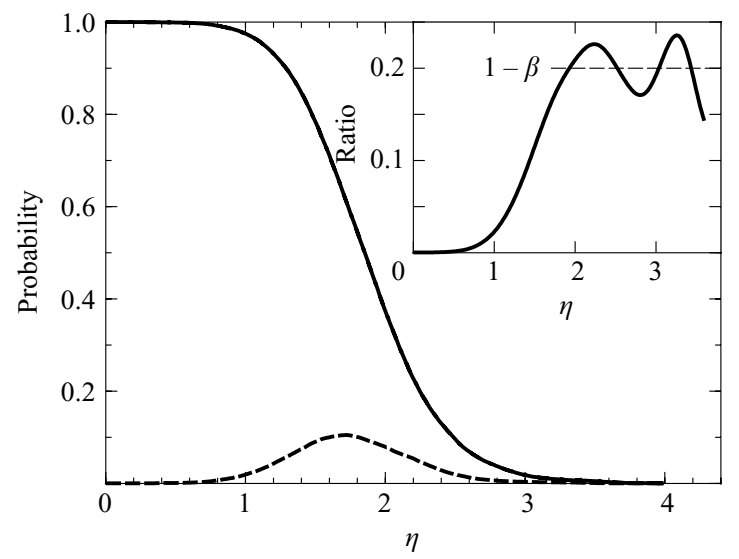

FIGURE 16. The probability to find jet fluid (- - and entrained fluid (- --$)$ as a function of the normalized distance $\eta=y / b_{u}(x)$ from the jet centreline. The inset shows the ratio of the probability to find entrained fluid and the probability to find jet fluid.

The present data are limited to an ensemble of planar measurements through the jet centreline. Hence, the total jet mass is determined by first estimating the probability $p(x, y)$ for a given pixel location $(x, y)$ to be within the jet envelope and then integrating the probability under the assumption that it is rotationally invariant with respect to the jet axis; multiplication with $U_{c}$ yields an equivalent enclosed jet mass flux:

$$
\dot{Q}=\rho U_{c} b_{u}^{2} \cdot \int_{0}^{\infty} p(x, \eta) 2 \pi \eta \mathrm{d} \eta,
$$

with $\eta \equiv\left(y-y_{0}\right) / b_{u}(x)$. Similarly, the probability $p_{e}$ for a given pixel location to be within the jet envelope and to contain irrotational fluid is determined. This probability is integrated in the way same as that in (4.2) and yields an estimate of the engulfed mass flux $\dot{Q}_{e}$. This indicates that the engulfed mass flux $\dot{Q}_{e}$ for the present data is only $8 \%$ of the total mass flux $\dot{Q}$ (Westerweel et al. 2005, figure $4 b$ ), which is consistent with the result found by Mathew \& Basu (2002). From these results it was concluded that engulfment does not appear to be the dominant process with respect to the entrainment of irrotational fluid.

When $p$ and $p_{e}$ are scaled with respect to the jet width $b_{u}$ they display a self-similar shape that is plotted in figure 16. The relative fraction of engulfed irrotational fluid decays rapidly away from the jet interface towards the jet centreline. The engulfed irrotational fluid occurs in a layer with a thickness that is approximately equal to the jet half-width. In our measurements $p_{e}$ retains a finite value of $1.5 \times 10^{-4}$ at the jet centreline, which indicates that a small fraction of irrotational fluid penetrates as far as the jet centreline, as has been reported by many others. The inset in figure 16 shows the ratio $p_{e} / p$ as a function of the distance from the centreline. The ratio is quite accurate near the jet centreline, but as both $p_{e}$ and $p$ vanish towards the outer jet region, the ratio shows some strong fluctuations. Nonetheless, one could say that this ratio of engulfed mass relative to the (turbulent) jet mass attains a more or less constant value of about 0.2. This implies a value of $\beta \approx 0.8$ in (2.20) and (2.24). As explained briefly in $\S 2$, in free shear layers the engulfment dominates the entrainment, resulting in significant volumes of irrotational flow within the turbulent flow region. 


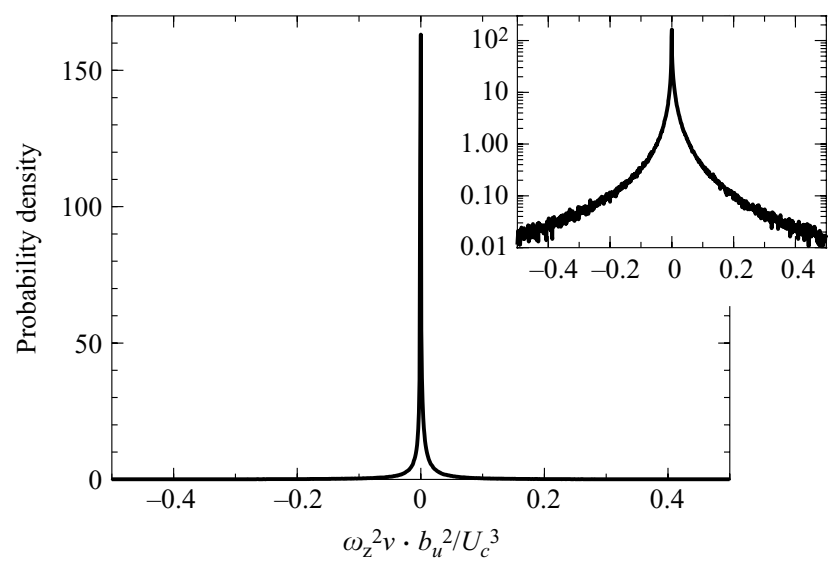

FIGURE 17. The p.d.f. for the enstrophy flux at the interface. The inset shows the same data in a semi-log plot.

\subsection{Enstrophy flux}

The analysis in $\$ 2.2$ demonstrated that a purely inertial transport of enstrophy at the interface should yield a finite value of the conditional enstrophy flux at the interface. The present combined PIV/LIF measurements only allow the measurement of the tangential component of the vorticity at the interface. It was shown by Bisset et al. (2002) that this is the dominant component of the vorticity. This was confirmed by volumetric measurements of Holzner et al. $(2007,2008)$ near the propagating turbulent/non-turbulent interface generated by an oscillating grid. Therefore the main term that contributes to the enstrophy flux would be $\left\langle v \omega_{z}^{2}\right\rangle$. This will be referred to as the 'enstrophy flux' in the remainder of this paper.

Figure 17 shows the p.d.f. of $v \omega_{z}^{2}$ at the jet interface. Note that the p.d.f. has a very narrow peak, which means that the enstrophy flux is highly intermittent. However, the p.d.f. is clearly symmetric (see inset of figure 17), which means that the ensemble mean value is zero; the mean enstrophy flux for the p.d.f. in figure 17 is $-0.003 U_{c}^{2} / b_{u}^{3}$. However, both positive and negative fluctuations in $v \omega_{z}^{2}$ can occur that have amplitudes that even exceed $0.5 U_{c}^{2} / b_{u}^{3}$.

Figure 18 shows the variation of $v \omega_{z}^{2}$ along the upper jet envelope of the data that corresponds to the jet depicted in figure 1. This graph is representative of all the instantaneous measurements in the data set. The enstrophy flux along the interface shows very strong intermittent behaviour, which is consistent with the shape of the p.d.f. for $v \omega_{z}^{2}$ in figure 17. It is noted that any large negative fluctuation is accompanied by a fluctuation of approximately equal amplitude but opposite sign a short distance downstream. For example, in figure 18 consider the pair of events at image locations 235 pixels and 350 pixels along the jet envelope (corresponding to $\left(x-x_{0}\right) / d=55.5$ and 60.7 respectively). Figure $19(a)$ shows the corresponding enlarged section of the LIF image with the in-plane velocity field and the detected jet interface overlayed. This graph shows that the paired event in figure 18 corresponds to an engulfment region at the jet interface.

Figure $19(b)$ shows the instantaneous vorticity $\omega_{z}$ in the same flow region. The vortices that are associated with the engulfment only become visible in the velocity map when the appropriate advection velocity has been subtracted. For the vortex with positive vorticity this velocity is equal to about $0.24 U_{c}$ in the axial direction and $-0.02 U_{c}$ in the radial direction. Subtracting this velocity for the total vector map yields the result shown in figure 20 . 


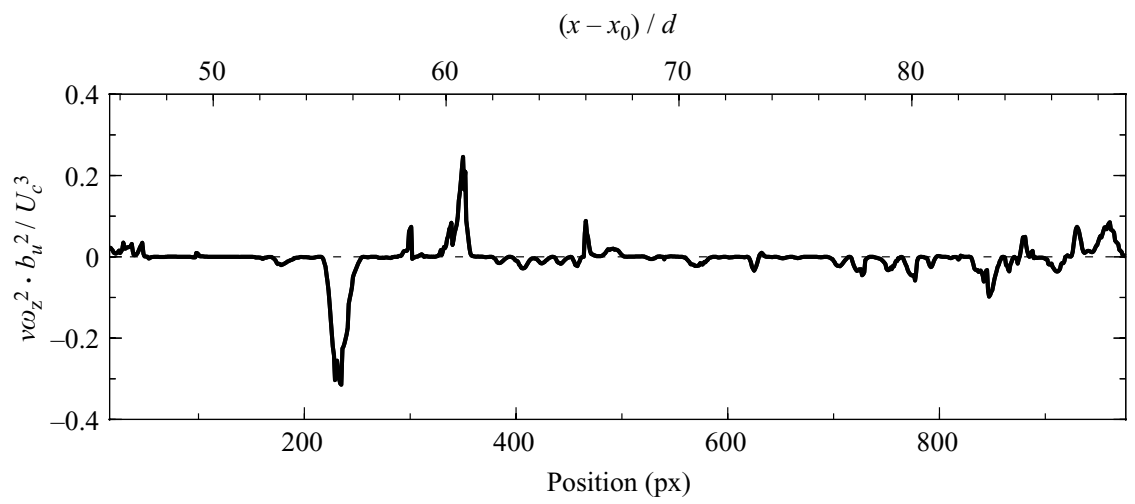

FIGURE 18. The instantaneous turbulent enstrophy transport $v \omega_{z}^{2}$ at the interface.

(a)

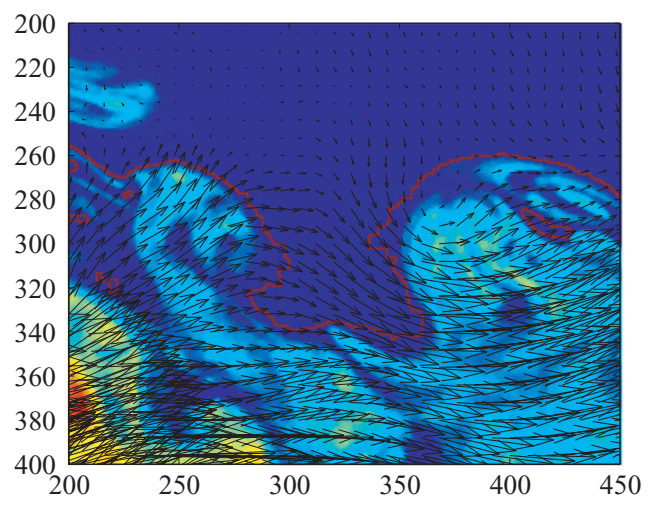

(b)

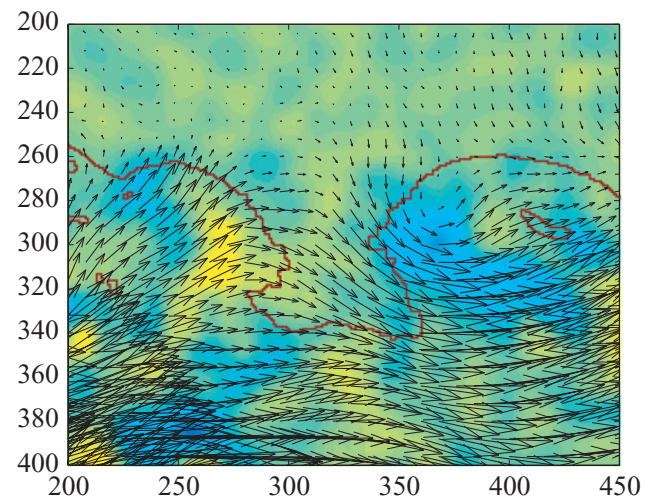

FIGURE 19. The instantaneous velocity field superimposed $(a)$ on the instantaneous concentration field and $(b)$ on the instantaneous vorticity field. The solid line represents the jet envelope.

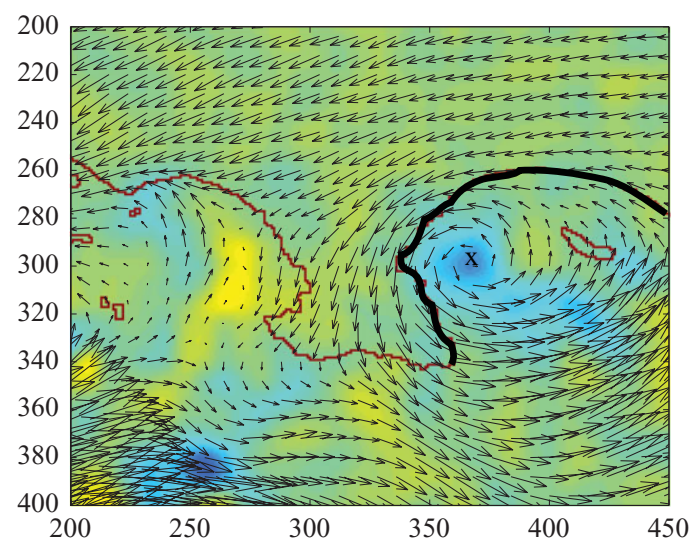

FIGURE 20. As in figure 19(b) but now with respect to a frame of reference that moves with the right vortex. Along the emphasized section of the interface the fluid motion induced by the right vortex $(x)$ is parallel to the interface. 


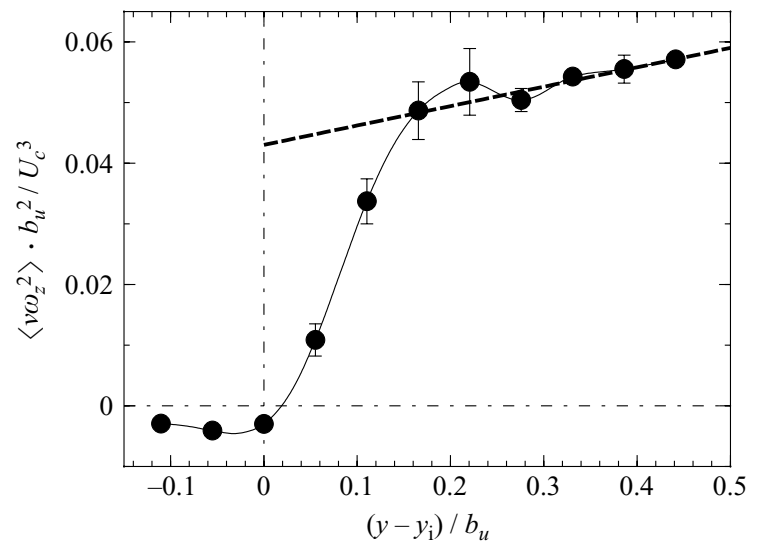

FIGURE 21. The conditional turbulent enstrophy flux $\left\langle v \omega_{z}^{2}\right\rangle$ as a function of the distance from the interface. The data points represent the PIV data spacing. The error bars represent the difference between the results for the upper and lower boundaries.

Note that in figure 19 turbulent jet fluid appears to be transported across the interface. However, in the frame of reference relative to the counterclockwise-rotating vortex, the velocity vectors are almost parallel to the jet interface. Hence the fluid motion that is associated with the vortices that entrain fluid by means of engulfment do not contribute to the transfer of irrotational fluid mass across the jet interface but continuously stretch the interface to keep it thin.

Since the engulfment event is associated with fluctuations in $v \omega_{z}^{2}$ of approximately equal amplitude but opposite sign, the net contribution of the engulfment event to the total enstrophy flux is small, if not negligible. In terms of the analysis in $\S 2.1$ this implies that these engulfment events do not contribute to $E_{b}$. This is in agreement with the previous observation that the engulfed fluid makes only a small contribution to the total jet mass flux.

In figure 21 the conditional turbulent enstrophy flux $\left\langle v \omega_{z}^{2}\right\rangle$ is plotted as a function of the distance from the interface. These results were obtained by averaging $v \omega_{z}^{2}$ along lines parallel to the jet envelope detected from a combination of all the PIV/LIF measurements. The results for the upper and lower envelopes were computed separately. The data points represent the average of the corresponding results from the upper and lower envelopes, with the error bars representing the difference between the results. The spacing of the data points in figure 21 corresponds to the 8 pixel data spacing of the PIV measurement. It should be noted that uncorrected results for the enstrophy were used (see figure 14); as a result the conditional enstrophy flux on the irrotational side of the interface retains a small negative value for the enstrophy flux which is the product of the mean square random error in the vorticity and the mean radial velocity.

On the turbulent side of the interface the enstrophy flux shows a jump in which it increases from a near-zero value at the interface to about $0.05 U_{c}^{3} / b_{u}^{2}$ over a distance of about $0.16 b_{u}$. The width over which the jump in $\left\langle v \omega_{z}^{2}\right\rangle$ occurs covers four data points (and is therefore greater than the spatial resolution of the PIV data); this value is approximately equal to the estimated and measured Taylor microscale (Westerweel et al. 2005; Ewing et al. 2007). This width of the layer corresponds to the prediction from the analysis by Hunt et al. (2006). For a distance of more than $0.16 b_{u}$ the conditional enstrophy flux increases at a much lower rate with increasing distance 
from the interface. The dashed line in figure 21 has been fitted to the data points, and extrapolating this line to the interface yields a virtual value of $0.043 U_{c}^{3} / b_{u}^{2}$. Given that $\left\langle\omega_{z}^{2}\right\rangle \approx 0.9$ and $\left\langle v^{2}\right\rangle^{1 / 2} \approx 0.10$ (see figure 14), it is found through (2.16) that $\alpha_{\text {turb }}=0.48$. This is consistent with the value $\alpha_{\text {turb }}=0.5$ based on our simple concept of nibbling in $\S 2.2$. The value for $\alpha_{\mathrm{sh}}$ is determined by $\langle u v\rangle \approx 0.007 U_{c}^{2}$ and $\Delta\left\langle q^{2}\right\rangle$, which is estimated at $(3 / 2) \times(0.08)^{2} U_{c}^{2}=0.01 U_{c}^{2}$ (figure 14). We thus find that $\alpha_{\mathrm{sh}}$ is 0.7 . This should be considered as an upper value; note that the value is considerably smaller, or even vanishes, in the absence of a mean shear. Given our previous result for $\Delta U / U_{c}$ between 0.06 and $0.09(\S 4.2)$, we obtain by means of (2.16) that $E_{b}$ is between $-0.1 U_{c}$ and $-0.08 U_{c}$. This close to our previous estimate of $E_{b} \approx-0.07 U_{c}$.

\subsection{Scalar transport}

The principles of the superlayer jump condition that apply to the axial momentum should also apply to the scalar concentration. The existence of a jump in the concentration has been documented in previous experiments (e.g. Mungal \& Hollingsworth 1989) and numerical simulations, and it is commonly accepted for unbounded turbulent flows. In principle the ratio of the conditional radial scalar flux and the jump in the conditional mean concentration should give the same value of the entrainment velocity $E_{b}$. However, as explained in $\S 3$, our measurements of the scalar flux in the present work are clearly under-resolved, which leads to an inaccurate estimate of $E_{b}$.

Since the entrainment velocity $E_{b}$ must be identical in both cases, an alternative approach was suggested in (2.7). Given the ratio of the eddy viscosity and eddy diffusivity, which is about $v_{e} / D_{e} \cong 0.5$ (Townsend 1976), the magnitude of the jump $\Delta U$ can be estimated from the mean conditional velocity and concentration. The jump $\Delta C$ and the variations of the velocity $\Delta U_{S}$ and concentration $\Delta C_{S}$ in the adjustment layer can be determined from the profiles in figure 13. Hence, it is found for the velocity $U_{S} \cong 0.9 U_{c}$, and for the concentration $\Delta C \cong 0.18 C_{c}$ and $C_{S} \cong 0.8 C_{c}$. Here the jump in the concentration $\Delta C$ is determined by extrapolating the linear profile in the turbulent flow region (see figure 13). Substitution of these numbers into (2.7) gives $\Delta U \cong 0.1 U_{c}$, which is in agreement with experimental result of $\Delta U=0.09 U_{c}$ determined in $\S 4.1$. Hence, the measurement of the scalar jump can be used to obtain an estimate of the jump in the momentum.

If a value of $\Delta C=0.3 C_{c}$ is used, then a much higher value for $\Delta U$ is obtained. It is noted that the value $v_{e} / D_{e} \cong 0.5$ is a 'bulk' value that may not be valid in the vicinity of the interface. When $\Delta U=0.09 U_{c}, U_{S}=0.9 U_{c}, \Delta C=0.3 C_{c}$ and $C_{S}=0.8 C_{c}$ are substituted in (2.7), then a ratio $v_{e} / D_{e} \cong 0.23$ is found. Clearly, the mixing layer at the interface affects the numerical value of $v_{e} / D_{e}$. This is evident in the present data in which close to the interface $\partial\langle C\rangle / \partial n \rightarrow 0$, which indicates a divergence of $D_{e}$. In addition, it is noted that the analysis in $\S 2.1$ applies to a planar interface. (Vortical eddy analysis following Hunt (1987) indicates that the ratio $v_{e} / D_{e}$ is reduced for curved shear layers.)

It was explained in $\S 2.3$ how the convolution of the p.d.f. of the interface position with the profiles of the conditional flow statistics can be used to reconstruct the mean profiles in a fixed laboratory reference frame. The p.d.f. of the interface position $p\left(y_{\mathrm{i}}\right)$ is modelled as a normal distribution with mean $\overline{y_{\mathrm{i}}} / b_{u}=1.93$ and a width $\sigma_{\mathrm{i}} / b_{u}=0.41$ (see figure 11). This is convoluted with a convenient model description of the conditional profile. The convolution of the p.d.f. for the position of the interface and the conditional mean vorticity profile yields the mean vorticity profile 


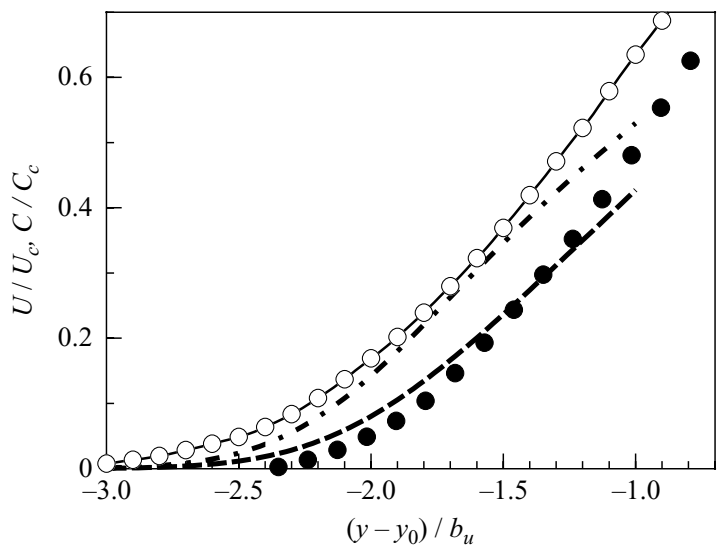

FiguRE 22. The reconstructed profiles of the normalized mean velocity $U / U_{c}(---)$ and the normalized mean scalar concentration $C / C_{c}(-\cdot-)$ as functions of the normalized distance from the centreline $\left(y-y_{0}\right) / b_{u}$. Results are obtained by taking the convolution of the p.d.f. of the interface position $p\left(y_{\mathrm{i}}\right)$ and the approximate expressions for the mean conditional velocity $\langle U\rangle$ and scalar concentration $\langle C\rangle$ shown in (2.26). The symbols represent the experimental data for $U / U_{c}$ (in figure 6) and $C / C_{c}$.

$\Omega_{z}(\cong-\partial \bar{U} / \partial x)$ in the laboratory reference frame. This was used by Westerweel et al. (2005) to determine whether the peak in the mean conditional vorticity profile makes a significant contribution to $\Omega_{z}$. For this purpose the p.d.f. was convolved with the functions $A$ and $A+B$ in (2.20). The value of $\beta$ was determined from figure 16, where it is found that $\beta=0.8$ for $\eta=1.93$. The experimental data agrees with the result of the convolution of the conditional mean profile with the p.d.f. of the interface position obtained when the peak in the conditional mean vorticity, represented by the interface term $B$, is included.

When a similar approach is followed for the conditional momentum flux, the ratio of $\overline{u v}$ and $\partial \bar{U} / \partial y$ yields the eddy viscosity in the outer region of the jet. This explains why a finite value for the eddy viscosity can exist in the outer region of the jet, despite the fact that the turbulence vanishes. Westerweel et al. (2005) obtained a value of $v_{e}=0.013 U_{c} b_{u}$. This value is about one half of the eddy viscosity of $v_{e}=0.031 U_{c} b_{u}$ in the bulk of the jet (see $\S 3$ ). This validates the estimated ratio of 0.5 for the eddy viscosity near the interface and in the bulk of the jet (see (2.22)).

Using the model descriptions for the profiles of $\langle U\rangle$ and $\langle C\rangle$ in (2.26) yields the results plotted in figure 22. Here we used the values $\partial\langle U\rangle / \partial n \cong 0.50 U_{c} / b_{u}$ and $\partial\langle C\rangle / \partial n \cong 0.42 C_{c} / b_{u}$ with $\beta=0.8$ as found in the experimental results. Note that the result in figure 22 is only valid near the mean interface position, i.e. $\left|y-y_{0}\right| \cong 2 b_{u}$. This implies that the predicted profiles for $U / U_{c}$ and $C / C_{c}$ do not approach unity towards the jet centreline. The predicted results for $U$ and $C$ are within reasonable agreement of the actual experimental data (Fukushima et al. 2002). Near the mean position of the interface (at $\left|y-y_{0}\right| / b_{u} \cong 2$ ) the profile for the mean scalar concentration $C$ is distinctively wider than the profile for the mean axial velocity $U$. The widths for which $C / C_{c}$ and $U / U_{c}$ reach a value of 0.3 and also for which the 'reconstructed' profiles for $U$ and $C$ begin to deviate from the experimental data) are $y=-1.62 b$ and $-1.32 b$ respectively. The ratio of these widths is 0.81 , which is very close to the ratio $b_{u} / b_{c}=0.77$ given in $\S 3$. Hence, the larger value of the jet half-width for the scalar concentration $b_{c}>b_{u}$ can be explained in terms of a larger jump $\Delta C / C_{c}$ in the concentration field in comparison with the jump $\Delta U / U_{c}$ in the axial momentum. 


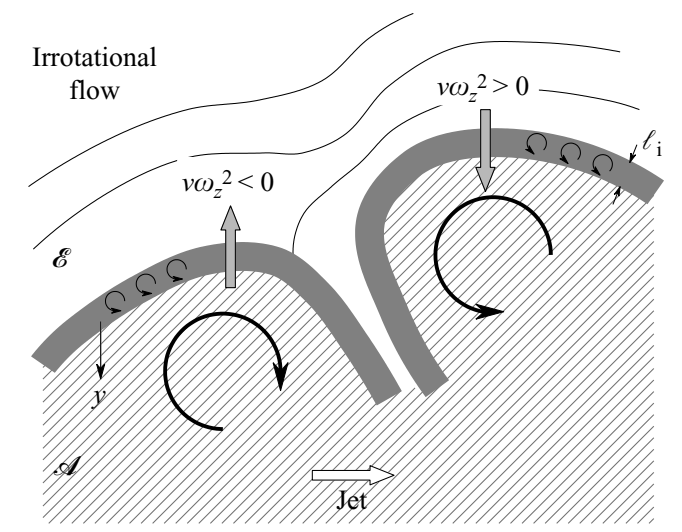

FIGURE 23. A schematic of the turbulent/non-turbulent interface, showing the sharp interface that is delineated by a thin (but weak) shear layer. Vortices (associated with engulfment events) strain the interface to maintain a thin interface layer.

\section{Conclusion}

This paper describes the experimental results using a combined PIV and LIF method to measure the entire velocity and scalar fields over space and time in a turbulent laboratory jet, extending the results of Westerweel et al. (2005). The experimental data, which are consistent with those of other experiments and with DNS, make it possible to identify and determine the physical and statistical properties of the flow near the fluctuating interface between rotational and irrotational fluctuations.

Firstly there is clear evidence for a continuous fluctuating boundary of the turbulent region, where the maxima of the conditional mean vorticity and scalar gradient are there. (The same sheet defines both these maxima.) The jumps $\Delta U$ in the velocity and $\Delta C$ in the scalar concentration across the sheet vary along the sheet, reflecting the interactions with the large eddy motions within the turbulence. (Thus the 'interface' description is quite consistent with the large eddy picture.)

Secondly the outward movement of the boundary, or entrainment process, characterized by the flux of vorticity is shown to be dominated by small-scale 'nibbling' turbulence generated at the interface, rather than by engulfing motion of large eddies. Because these only extend a limited distance into the turbulence, there is only very little unmixed external fluid in the interior of the turbulence. This is consistent with the conclusions of Mathew \& Basu (2002) and Hunt et al. (2008). A schematic summarizing the structure of the turbulent/non-turbulent interface is depicted in figure 23, showing large eddies (associated with an engulfment event) that strain the shear layer layer and thus maintain the sharpness of the interface. The analysis of the engulfing mechanism by Hunt et al. (2008) shows that the magnitude of engulfing events are relatively small when their streamwise length scale is less than the width of the shear layer. This is found in flows like jets and wakes with significant mean curvature, but not in plane free shear layers in which the lower mean curvature leads to larger scale eddy motion, that approximately spans the whole shear layer (cf. Reynolds 1972; Brown \& Roshko 1974).

The present experimental study and data analysis are limited to the case of a single and rather low value of the Reynolds number. Further experimental data at much higher Reynolds numbers following the present quantitative approach would 
be required to determine if either 'nibbling' or 'engulfment' becomes the dominant entrainment process in the limit of high Reynolds number when there is larger separation between large-scale and small-scale turbulent motions. Many of the reports that describe and explain entrainment in terms of the engulfment process have not been based on detailed flow measurements near the interface. However, the data of Mungal \& Hollingsworth (1989) and cloud studies at very high Reynolds numbers of the order of $10^{6}-10^{7}$ show that in jets and plumes, which are dominated by initial momentum and buoyancy, there is very little unmixed fluid in the interior. This would imply relatively weak engulfment, but this appears to change dramatically with externally or internally generated turbulence (Tsai et al. 2007; Burnet \& Brenguier 2007). Engulfment appears to be dominant in the case of a free plane shear layer or when jets, plumes or wakes are strongly perturbed by internal or external fluctuations. In that case the basic structure changes, and engulfment or detrainment dominates the entrainment process (Bhat \& Narasimha 1996; Gaskin, McKernan \& Xue 2004; Hunt et al. 2006; Khorsandi, Gaskin \& Mydlarski 2007). The present quantitative experimental approach may shed light on the entrainment process in other flows and for other flow conditions. The use of volumetric measurement methods would make it possible to define the interface based on the amplitude of the vorticity vector (e.g. Holzner et al. 2008) and would avoid complications in the present approach e.g. for the case of a low Schmidt number (or low Prandtl number when the scalar transport concerns the transport of heat.)

Furthermore, a comprehensive model for interface dynamics is still needed. Idealized models demonstrate some key aspects such as the persistence of the vortex sheet and the jump in the mean scalar. The internal dynamics of the vortex sheet, which determine the rate of entrainment by 'nibbling', have not been modelled, although its mean thickness has been estimated as equal to that of the Taylor microscale. The other component of entrainment, caused by engulfment, also needs to be modelled more explicitly, by extending the idealized modelling of Hunt et al. (2008) to analyse the rate of nibbling to engulfing entrainment in different flows and in the limit of high Reynolds number. Through improved modelling of these interface entrainment processes it should then be possible to account for the sudden decrease in entrainment and increase in intermittency caused by external turbulence and/or body forces within the jet (Agrawal \& Prasad 2002; Hunt et al. 2006; Tsai et al. 2007). Another characteristic feature of jets and wakes is the sensitivity to their initial condition, which is also likely to be related to the turbulent interface structure.

We are grateful for the suggestions and comments by the referees and by B. J. Boersma on an earlier version of this manuscript. This work was supported by the Foundation for Fundamental Research on Matter (www.fom.nl). JCRH acknowledges support from Cornell University, where he was a Mary B. Upson visiting professor.

\section{REFERENCES}

Adrian, R. J. 1991 Particle-imaging techniques for experimental fluid mechanics. Annu. Rev. Fluid Mech. 23, 261-304.

Agrawal, A. \& Prasad, A. K. 2002 Organizational modes of large-scale vortices in an axisymmetric turbulent jet. Flow Turbul. Combust. 68, 359-377.

Antonia, R. A. 1981 Conditional sampling in turbulence measurement. Annu. Rev. Fluid Mech. 13, $131-156$.

BHAT, G. S. \& NARASImHa, R. 1996 A volumetrically heated jet: large-eddy structure and entrainment characteristics. J. Fluid Mech. 325, 303-330. 
Bisset, D. K., Hunt, J. C. R., CAI, X. \& Rogers, M. M. 1998 Interfaces at the outer boundaries of turbulent motions. In Annual Research Briefs, pp. 125-135. Centre for Turbulence Research.

Bisset, D. K., Hunt, J. C. R. \& Rogers, M. M. 2002 The turbulent/non-turbulent interface bounding a far wake. J. Fluid Mech. 451, 383-410.

Boersma, B. J., Brethouwer, G. \& Nieuwstadt, F. T. M. 1998 A numerical investigation on the effect of the inflow conditions on the self-similar region of a round jet. Phys. Fluids 10, 899-909.

Broadwell, J. E. \& Breidenthal, R. E. 1982 A simple model of mixing and chemical reaction in a turbulent shear layer. J. Fluid Mech. 125, 397-410.

Brown, G. L. \& Roshko, A. 1974 On density effects and large structure in turbulent mixing layers. J. Fluid Mech. 64, 775-816.

Buch, K. A. \& DAHM, W. J. A. 1996 Experimental study of the fine-scale structure of conserved scalar mixing in turbulent flows. Part 1. Sc 》1. J. Fluid Mech. 317, 21-71.

Burnet, F. \& Brenguier, J.-L. 2007 Observational study of the entrainment-mixing process in warm convective clouds. J. Atmos. Sci. 64, 1995-2011.

CARruthers, D. J. \& Hunt, J. C. R. 1986 Velocity fluctuations near an interface between a turbulent region and a stably stratified layer. J. Fluid Mech. 165, 475-501.

Cazalbou, J. B., Spalart, P. R. \& Bradshaw, P. 1994 On the behaviour of two-equation models at the edge of a turbulent region. Phys. Fluids 6, 1797-1804.

Chevray, R. \& Tutu, N. K. 1978 Intermittency and preferential transport of heat in round jet. J. Fluid Mech. 88, 133-160.

Chua, L. P. \& Antonia, R. A. 1990 Turbulent Prandtl number in a circular jet. Intl J. Heat Mass Transfer 33, 331-339.

Corrsin, S. \& Kistler, A. L. 1955 Free-stream boundaries of turbulent flows. Tech Rep. 1244. NACA.

Dahm, W. J. A. \& Dimotakis, P. E. 1987 Measurements of entrainment and mixing in turbulent jets. AIAA J. 25, 1216-1223.

Dimotakis, P. E. 2000 The mixing transition in turbulent flows. J. Fluid Mech. 409, 69-98.

Ewing, D., Frohnapfel, B., George, W. K., Pedersen, J. M. \& Westerweel, J. 2007 Two-point similarity in the round jet. J. Fluid Mech. 577, 309-330.

Ferré, J. A., Mumford, J. C., Savill, A. M. \& Giralt, F. 1990 Three-dimensional largeeddy motions and fine-scale activity in a plane turbulent wake. J. Fluid Mech. 210, 371-414.

Fukushima, C., Aanen, L. \& Westerweel, J. 2002 Investigation of the mixing process in an axisymmetric turbulent jet using PIV and LIF. In Laser Techniques for Fluid Mechanics (Eds. R. J. Adrian, D. F. G. Durão, F. Durst, M. V. Heitor, M. Maeda \& J. Whitelaw), pp. 339-356. Springer.

Gaskin, S. J., McKernan, M. \& Xue, F. 2004 The effect of background turbulence on jet entrainment: an experimental study of a plane jet in a shallow coflow. J. Hydraul. Res. 42, 531-540.

Hernan, M. A. \& Jimenez, J. 1982 Computer analysis of a high-speed film of the plane turbulent mixing layer. J. Fluid Mech. 119, 323-345.

Hinze, J. O. 1975 Turbulence, 2nd edn. McGraw-Hill.

Holzner, M., Liberzon, A., Nikitin, N., Kinzelbach, W. \& Tsinober, A. 2007 Small-scale aspects of flows in proximity of the turbulent/nonturbulent interface. Phys. Fluids 19, 071702.

Holzner, M., Liberzon, A., Nikitin, N., Lühti, B., Kinzelbach, W. \& Tsinober, A. 2008 A Lagrangian investigation of the small-scale features of turbulent entrainment through particle tracking and direct numerical simulation. J. Fluid Mech. 598, 465-475.

Hunt, J. C. R. 1987 Vorticity and vortex dynamics in complex turbulent flows. Trans. Can. Soc. Mech. Engng 11, 21-35.

Hunt, J. C. R. \& Durbin, P. A. 1999 Perturbed vortical layers and shear sheltering. Fluid Dyn. Res. 24, 375-404.

Hunt, J. C. R., Eames, I. \& Westerweel, J. 2006 Mechanics of inhomogeneous turbulence and interfacial layers. J. Fluid Mech. 554, 499-519.

Hunt, J. C. R., EAmes, I. \& WesterweEl, J. 2008 Vortical interactions with interfacial shear layers. In Proc. of the IUTAM Symp. on Computational Physics and New Perspectives in Turbulence (ed. Y. Kaneda), vol. 4, pp. 331-338. Springer. 
Hunt, J. C. R., Sandham, N. D., Vassilicos, J. C., Launder, B. E., Monkewitz, P. A. \& Hewitt, G. F. 2001 Developments in turbulence research: a review based on the 1999 programme of the Isaac Newton Institute, Cambridge. J. Fluid Mech. 436, 353-391.

Hussain, A. K. M. F. \& ClaRK, A. R. 1981 On the coherent structure of the axisymmetric mixing layer: a flow-visualization study. J. Fluid Mech. 104, 263-294.

Keane, R. D. \& Adrian, R. J. 1992 Theory of cross-correlation analysis of PIV images. Appl. Sci. Res. 49, 191-215.

Khorsandi, B., Gaskin, S. \& Mydlarski, L. 2007 Effect of background turbulence on the velocity field of a turbulent jet. In Proc. of the 5th Intl Symp. on Environmental Hydraulics (ISEH V), Tempe, AZ.

Kibens, V., Kovasznay, L. S. G. \& Oswald, L. J. 1974 Turbulent-nonturbulent interface detector. Rev. Sci. Instrum. 45, 1138-1144.

Kovasznay, L. S. G., Kibens, V. \& Blackwelder, R. F. 1970 Large-scale motion in the intermittent region of a turbulent boundary layer. J. Fluid Mech. 41, 283-325.

LANDRETH, C. C. \& ADRIAN, R. J. 1990 Impingement of a low Reynolds number turbulent circular jet onto a flat plate at normal incidence. Exp. Fluids 9, 74-84.

Lubbers, C. L., Brethouwer, G. \& Boersma, B. J. 2001 Simulation of the mixing of a passive scalar in a round turbulent jet. Fluid Dyn. Res. 28, 189-208.

L'vov, V. S., Pomyalov, A., Procaccia, I. \& Govindarajan, R. 2008 Random vortex-street model for a self-similar plane turbulent jet. Phys. Rev. Lett. 101, 094503.

Mathew, J. \& Basu, A. 2002 Some characteristics of entrainment at a cylindrical turbulence boundary. Phys. Fluids 14, 2065-2072.

Mungal, M. G. \& Hollingsworth, D. K. 1989 Organized motion in a very high Reynolds number jet. Phys. Fluids A 1, 1615-1623.

Mungal, M. G., Karasso, P. S. \& Lozano, A. 1991 The visible structure of turbulent jet diffusion flames: large-scale organization and flame tip oscillation. Combust. Sci. Technol. 76, $165-185$.

Panchapakesan, N. R. \& Lumley, J. L. 1993 Turbulence measurements in axisymmetric jets of air and helium. Part 1. Air jet. J. Fluid Mech. 246, 197-223.

Phillips, O. M. 1955 The irrotational motion outside a free boundary layer. Proc. Camb. Phil. Soc. 51, 220.

Pope, S. B. 2000 Turbulent Flows. Cambrige University Press.

Prandtl, L. 1956 Essentials of Fluid Dynamics. Dover.

Prasad, R. R. \& SReenivasan, K. R. 1989 Scalar interfaces in digital images of turbulent flows. Exp. Fluids 7, 259-264.

Raffel, M., Willert, C. \& Kompenhans, J. 1998 Particle Image Velocimetry: A Practical Guide. Springer.

ReYnolds, W. C. 1972 Large-scale instabilities of turbulent wakes. J. Fluid Mech. 54, 481-488.

Ruban, A. I. \& Vonatsos, K. N. 2008 Discontinuous solutions of the boundary-layer equations. J. Fluid Mech. 614, 407-424.

Sandham, N. D., Mungal, M. G., Broadwell, J. E. \& Reynolds, W. C. 1988 Scalar entrainment in the mixing layer. In Proc. of the CTR Summer Program, pp. 69-76.

DA Silva, C. B. \& Pereira, J. C. F. 2008 Invariants of the velocity-gradient, rate-of-strain, and rate-of-rotation tensors across the turbulent/nonturbulent interface in jets. Phys. Fluids 20, 055101.

Stanislas, M., Okamoto, K., Kähler, C. J. \& Westerweel, J. 2005 Main results of the Second International PIV Challenge. Exp. Fluids 39, 170-191.

Strang, E. J. \& Fernando, H. J. S. 2001 Vertical mixing and transports through a stratified shear layer. J. Phys. Oceanogr. 31, 2026-2048.

Townsend, A. A. 1976 The Structure of Turbulent Shear Flow, 2nd edn. Cambridge University Press.

Tropea, C., Yarin, A. \& Foss, J. (Ed.) 2007 Handbook of Experimental Fluid Mechanics. Springer.

Tsai, Y. S., Hunt, J. C. R., Nieuwstadt, F. T. M., Westerweel, J. \& Gunasekaran, B. P. N. 2007 Effect of strong external turbulence on a wall jet boundary layer. Flow Turbul. Combust. 79, $155-174$.

TsinOBer, A. 2001 An Informal Introduction to Turbulence. Kluwer. 
Turner, J. S. 1986 Turbulent entrainment. J. Fluid Mech. 173, 431-471.

WALKER, D. A. 1987 A fluorescent technique for measurement of concentration in mixing liquids. J. Phys. E 20, 217-224.

Wallace, J. M. \& Foss, J. F. 1995 The measurement of vorticity in turbulent flows. Annu. Rev. Fluid Mech. 27, 469-514.

Webster, D. R., Roberts, P. J. W. \& RA'Ad, L. 2001 Simultaneous DPTV/PLIF measurements of a turbulent jet. Exp. Fluids 30, 65-72.

WeSTERWEEL, J. 1994 Efficient detection of spurious vectors in particle image velocimetry data sets. Exp. Fluids 16, 236-247.

WesterweEl, J. 2000 Theoretical analysis of the measurement precision in particle image velocimetry. Exp. Fluids 29, S3-12.

Westerweel, J., Dabiri, D. \& Gharib, M. 1997 The effect of a discrete window offset on the accuracy of cross-correlation analysis of digital PIV recordings. Exp. Fluids 23, 20-28.

Westerweel, J., Fukushima, C., Pedersen, J. M. \& Hunt, J. C. R. 2005 Mechanics of the turbulent/nonturbulent interface of a jet. Phys. Rev. Lett. 95, 174501.

Westerweel, J., Hofmann, T., Fukushima, C. \& Hunt, J. C. R. 2002 The turbulent/non-turbulent interface at the outer boundary of a self-similar turbulent jet. Exp. Fluids 33, 873-878.

Westerweel, J. \& Scarano, F. 2005 Universal outlier detection for PIV data. Exp. Fluids 39, 1096-1100.

WiLLERT, C. 1997 Stereoscopic digital particle image velocimetry for application in wind tunnel flows. Meas. Sci. Technol. 8, 1465-1479.

Wygnanski, I. \& Fiedler, H. E. 1969 Some measurements in the self-preserving jet. J. Fluid Mech. 38, 577-612.

Yoda, M., Hesselink, L. \& Mungal, M. G. 1994 Instantaneous three-dimensional concentration measurements in the self-similar region of a round high-Schmidt-number jet. J. Fluid Mech. 279, 313-350. 